\title{
Shillapoo Wildlife Area 2007 Follow-up HEP Report
}

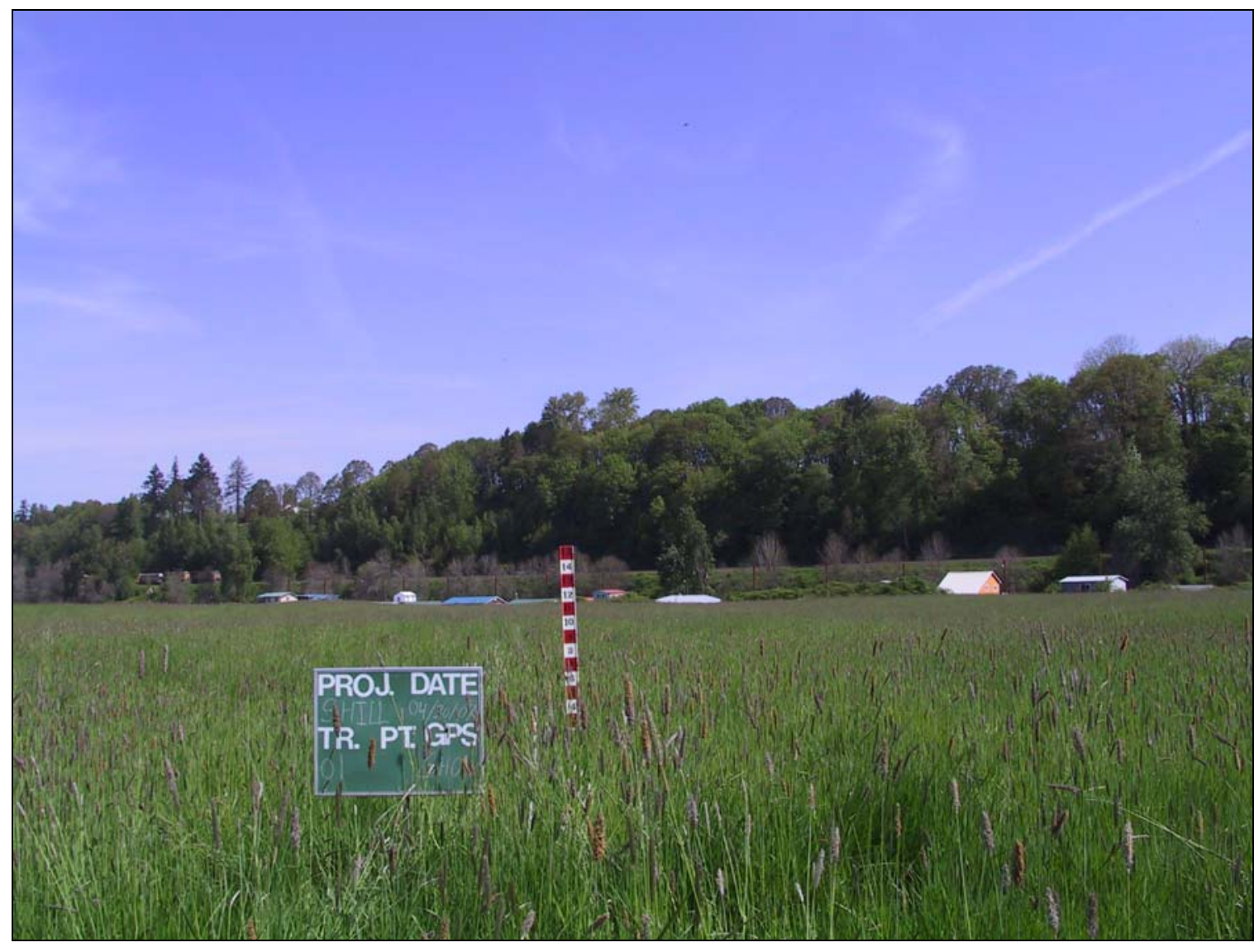

Compiled By

Paul R Ashley - CBFWA

For

Joe DeHerrera - BPA

And

Nathan Pamplin - WDFW

March 2008 


\section{Table of Contents}

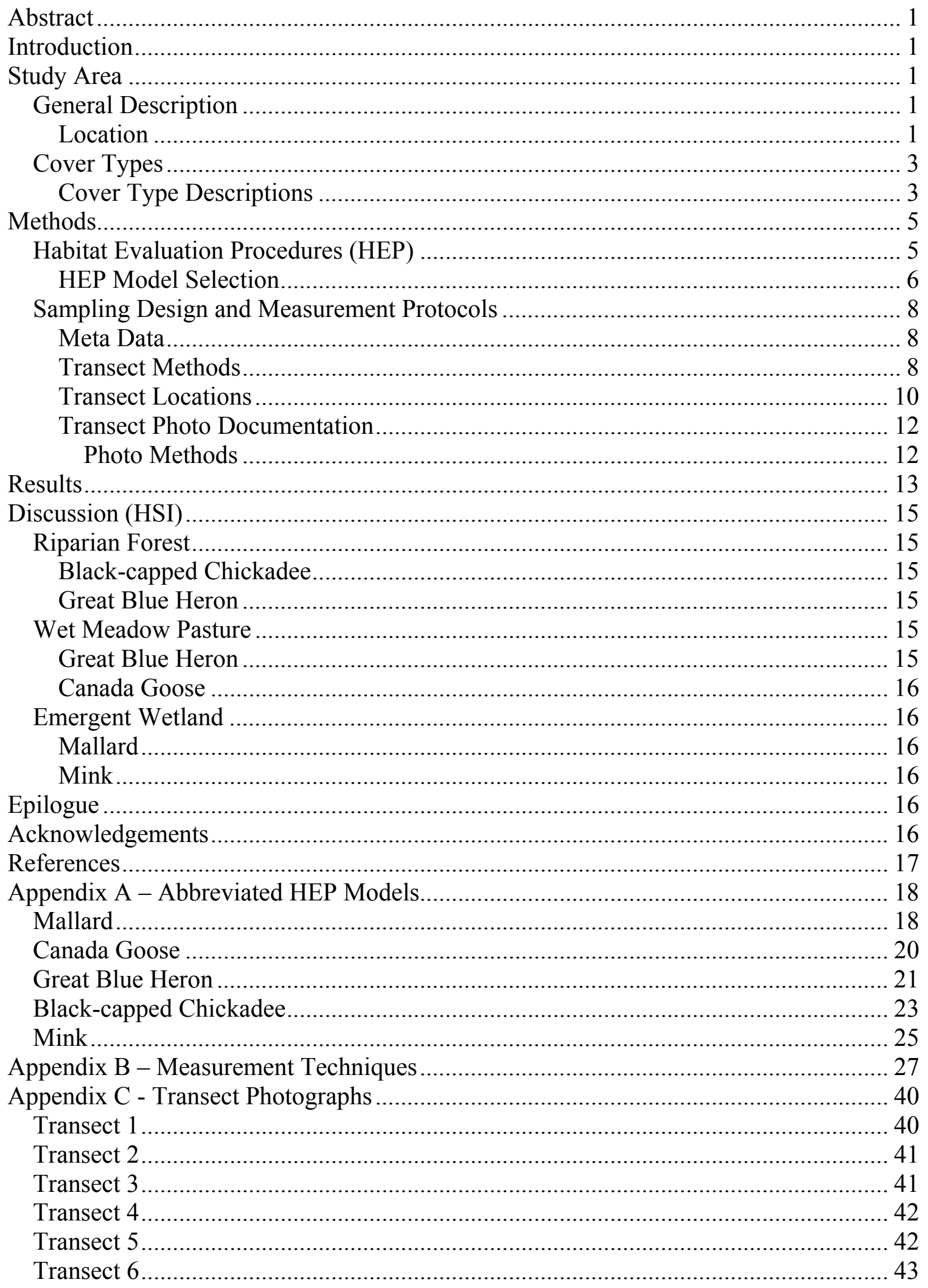




\section{Shillapoo WA 2007 HEP Report}

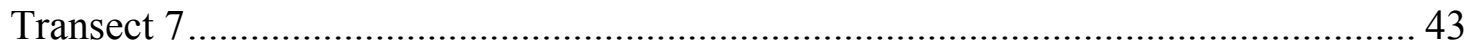

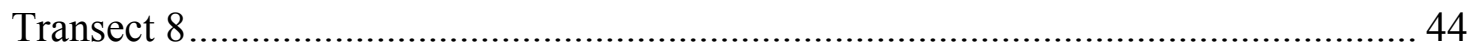

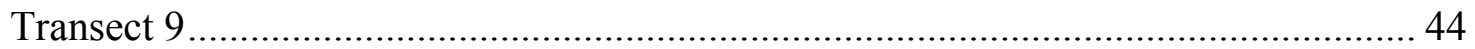

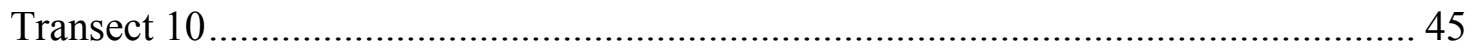

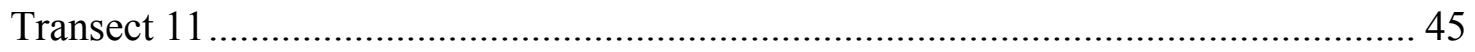

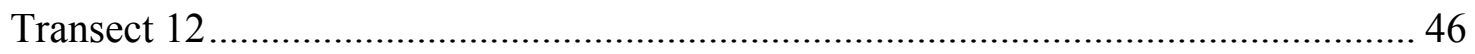

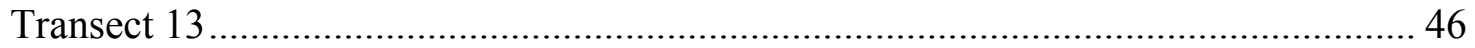

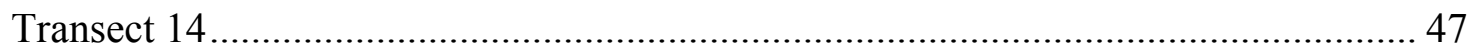

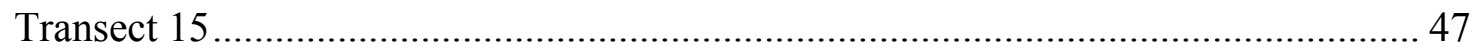

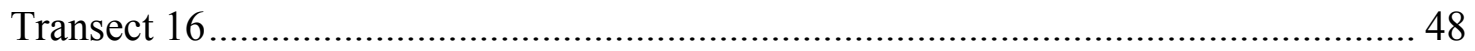

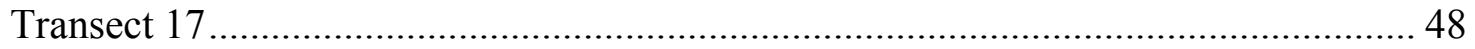

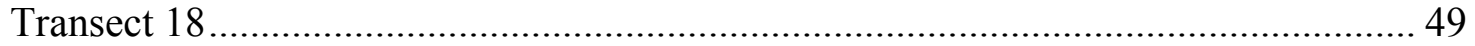




\section{Table of Figures}

Figure 1. Shillapoo Wildlife Area location and Egger and Herzog parcels...................... 2

Figure 2. Area surveyed during 2007 follow-up HEP survey analysis (Egger and Herzog

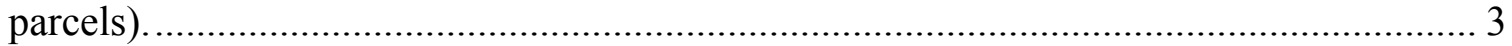

Figure 3. Wet meadow pasture cover type example................................................... 4

Figure 4. Riparian forest cover type example....................................................... 5

Figure 5. HEP data collection and processing flow chart........................................ 9

Figure 6. Follow-up HEP transect location start (S) and end (E) points (north half of

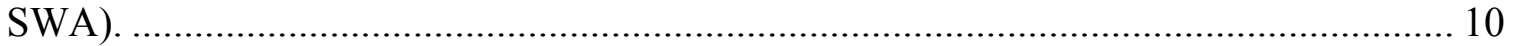

Figure 7. Follow-up HEP transect location start (S) and end (E) points (south half of

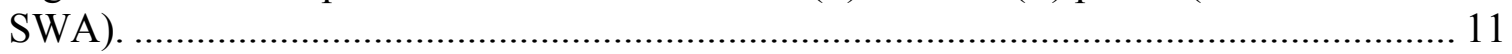

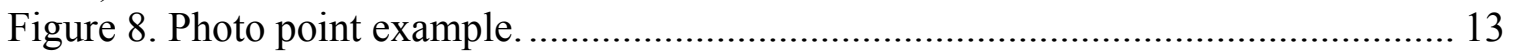




\section{List of Tables}

Table 1. Shillapoo Wildlife area cover type definitions................................................. 4

Table 2. Habitat suitability index verbal equivalency table.............................................. 6

Table 3. Shillapoo Wildlife Area 2007 follow-up HEP cover type/species matrix............. 6

Table 4. Bonneville Dam loss assessment matrix.......................................................... 7

Table 5. John Day Dam loss assessment matrix........................................................ 7

Table 6. Shillapoo Wildlife Area 2007 follow-up HEP transect coordinates, azimuths, and

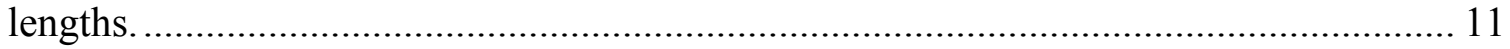

Table 7. Shillapoo Wildlife Area 2007 HEP results summary........................................ 14

Table 8. Follow-up and baseline habitat suitability index comparison summary............. 15 


\section{Abstract}

In April and May 2007 the Regional HEP Team (RHT) conducted a follow-up HEP analysis on the Egger (612 acres) and Herzog (210 acres) parcels located at the north end of the Shillapoo Wildlife Area. The Egger and Herzog parcels have been managed with Bonneville Power Administration funds since acquired in 1998 and 2001 respectively.

Slightly more than 936 habitat units (936.47) or 1.14 HUs per acre was generated as an outcome of the 2007 follow-up HEP surveys. Results included 1.65 black-capped chickadee HUs, 280.57 great blue heron HUs, 581.45 Canada goose HUs, 40 mallard HUs, and 32.80 mink HUs.

\section{Introduction}

A follow-up Habitat Evaluation Procedures (HEP) (USFWS 1980) analysis was conducted by the Columbia Basin Fish and Wildlife Authority's (CBFWA) Regional HEP Team (RHT) during April and May 2007 to document changes in habitat quality and to determine the number of habitat units (HUs) to credit Bonneville Power Administration (BPA) for providing operation and maintenance (O\&M) funds since WDFW acquired the parcels. The 2007 follow-up HEP evaluation was limited to Shillapoo Wildlife Area (SWA) parcels purchased with Bonneville Power Administration funds.

D. Budd (pers. comm.) reported WDFW purchased the 612 acre Egger Farms parcel on November 2, 1998 for $\$ 1,737,000^{1}$ and the 210 acre Herzog acquisition on June 21, 2001 for $\$ 500,000$ with Memorandum of Agreement funds (BPA and WDFW 1996) as partial fulfillment of BPA's wildlife mitigation obligation for construction of Bonneville and John Day Dams (Rasmussen and Wright 1989). Anticipating the eventual acquisition of the Egger and Herzog properties, WDFW conducted HEP surveys on these lands in 1994 to determine the potential number of habitat units to be credited to BPA. As a result, HEP surveys and habitat unit calculations were completed as much as seven years prior to acquiring the sites.

The term "Shillapoo Wildlife Area" will be used to describe only the Herzog and Egger parcels in this document. Details and results of the HEP analysis are included in this report.

\section{Study Area}

\section{General Description}

\section{Location}

The SWA is located in southwest Washington approximately two miles northwest of Vancouver, Washington (Figure 1). The Columbia River lies immediately to the west while Vancouver Lake is approximately 0.25 miles to the southeast. General area Universal Trans Mercator (UTM) coordinates are 10U 0521540E, 5061386N (red “star" in Figure 1).

\footnotetext{
${ }^{1}$ Joe DeHerrera (BPA) stated BPA records indicate that the Egger Farms parcel cost $\$ 1,740,000$.
} 


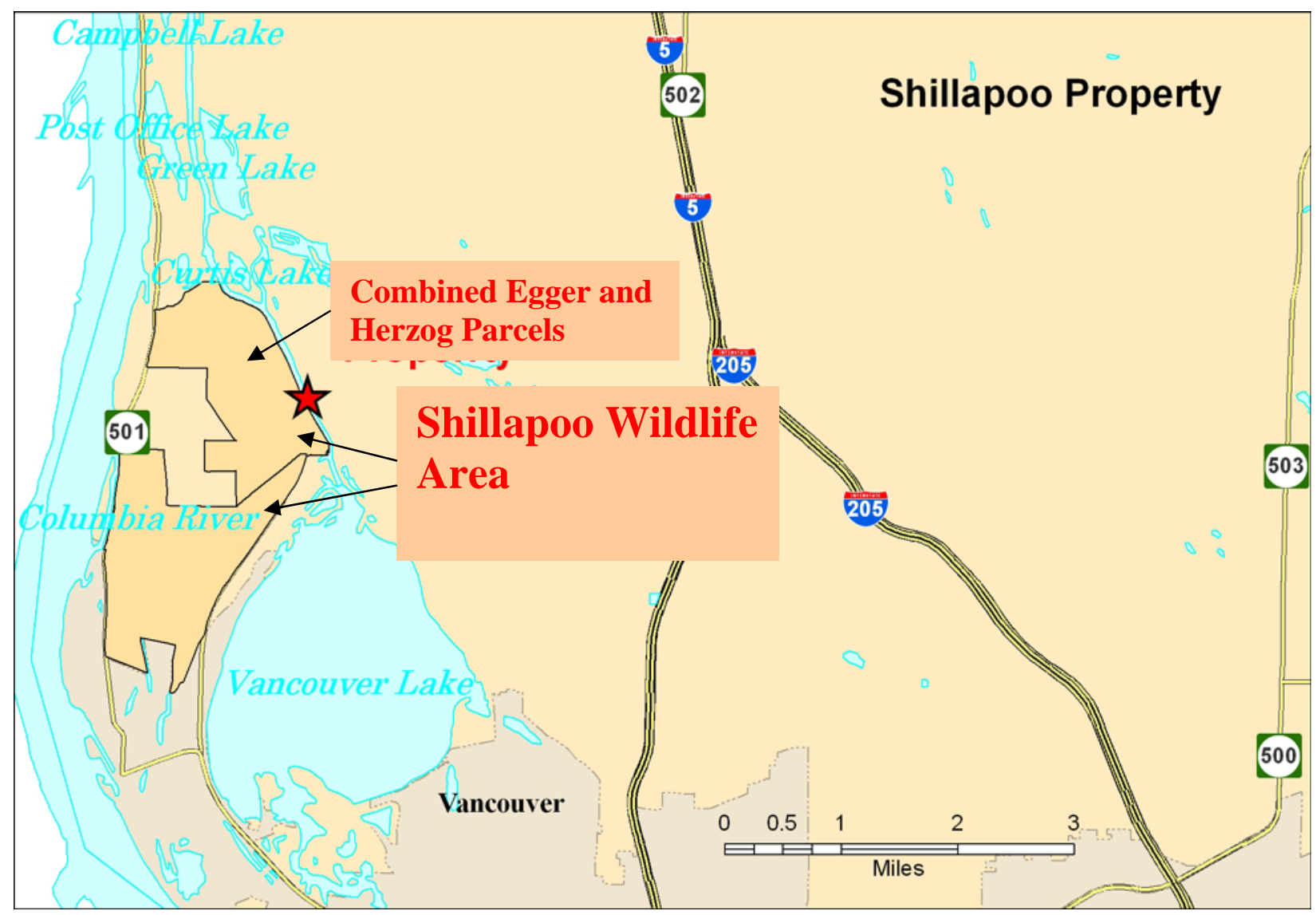

Figure 1. Shillapoo Wildlife Area location and Egger and Herzog parcels.

Regional HEP Team staff used hard copy maps provided by WDFW (B. Calkins, pers. comm.) to construct the boundary map illustrated in Figure 2 (Maptech ${ }^{\circledR}$ mapping software). As a result, map boundaries are approximate locations. Only the Egger and Herzog parcels acquired with BPA funds are shown in Figure 2 (these parcels represent approximately the north half of the entire SWA shown in Figure 1). 


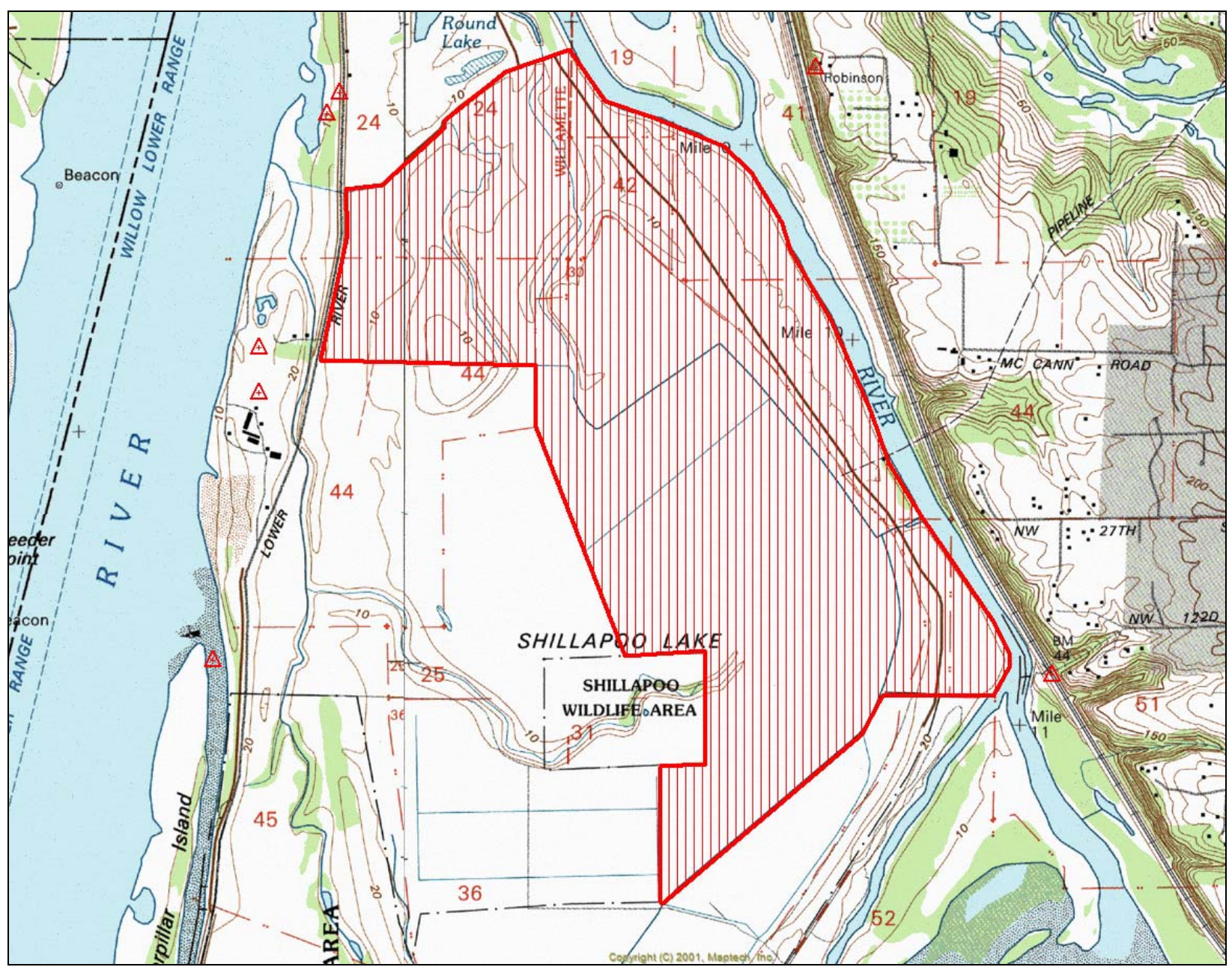

Figure 2. Area surveyed during 2007 follow-up HEP survey analysis (Egger and Herzog parcels).

\section{Cover Types}

Shillapoo Wildlife Area cover types transitioned from primarily active agriculture to wet meadow pasture and emergent wetland cover types since baseline HEP surveys were completed in 1994. The wet meadow cover type replaced the active agriculture cover type by 2007. Additional emergent wetland enhancements are planned and will likely be in place prior to the next follow-up HEP analysis (B. Calkins, pers. comm.).

General cover type descriptions and maps were provided by SWA staff (B. Calkins, pers. comm.). Three primary cover types were evaluated by Regional HEP Team staff i.e., riparian forest ( 5 acres), wet meadow pasture ( 777 acres), and emergent wetland (40 acres) for a total of 822 acres.

\section{Cover Type Descriptions}

The wet meadow pasture cover type dominated the landscape comprising approximately $95 \%$ of the area while emergent wetlands comprised less than $5 \%$. Trace amounts of riparian forest $(<1 \%$ of the SWA) were also present. Cover types are defined in Table 1. 
Shillapoo WA 2007 HEP Report

Table 1. Shillapoo Wildlife area cover type definitions.

\begin{tabular}{|l|c|l|}
\hline \multicolumn{1}{|c|}{ Cover Type } & Acres & \multicolumn{1}{c|}{ Description } \\
\hline Wet Meadow Pasture & 777 & $\begin{array}{l}\text { Dominated by mesic herbaceous vegetation. Shrub cover }< \\
5 \% .\end{array}$ \\
\hline Emergent Wetland & 40 & $\begin{array}{l}\text { Palustrine sites with }>5 \% \text { emergent vegetation. } \\
\text { Riparian Forest }\end{array}$ \\
\hline Total & $\mathbf{8 2 2}$ & $\begin{array}{l}\text { This cover type is dominated by hydrophytic/deciduous trees } \\
\text { and shrubs. Associated with lentic/lotic systems. }\end{array}$ \\
\hline
\end{tabular}

Examples of wet meadow pasture and riparian forest cover types are shown in Figure 3 and Figure 4 respectively (an emergent wetland photograph was not available). Review specific transect results including shrub/tree species information at data link.

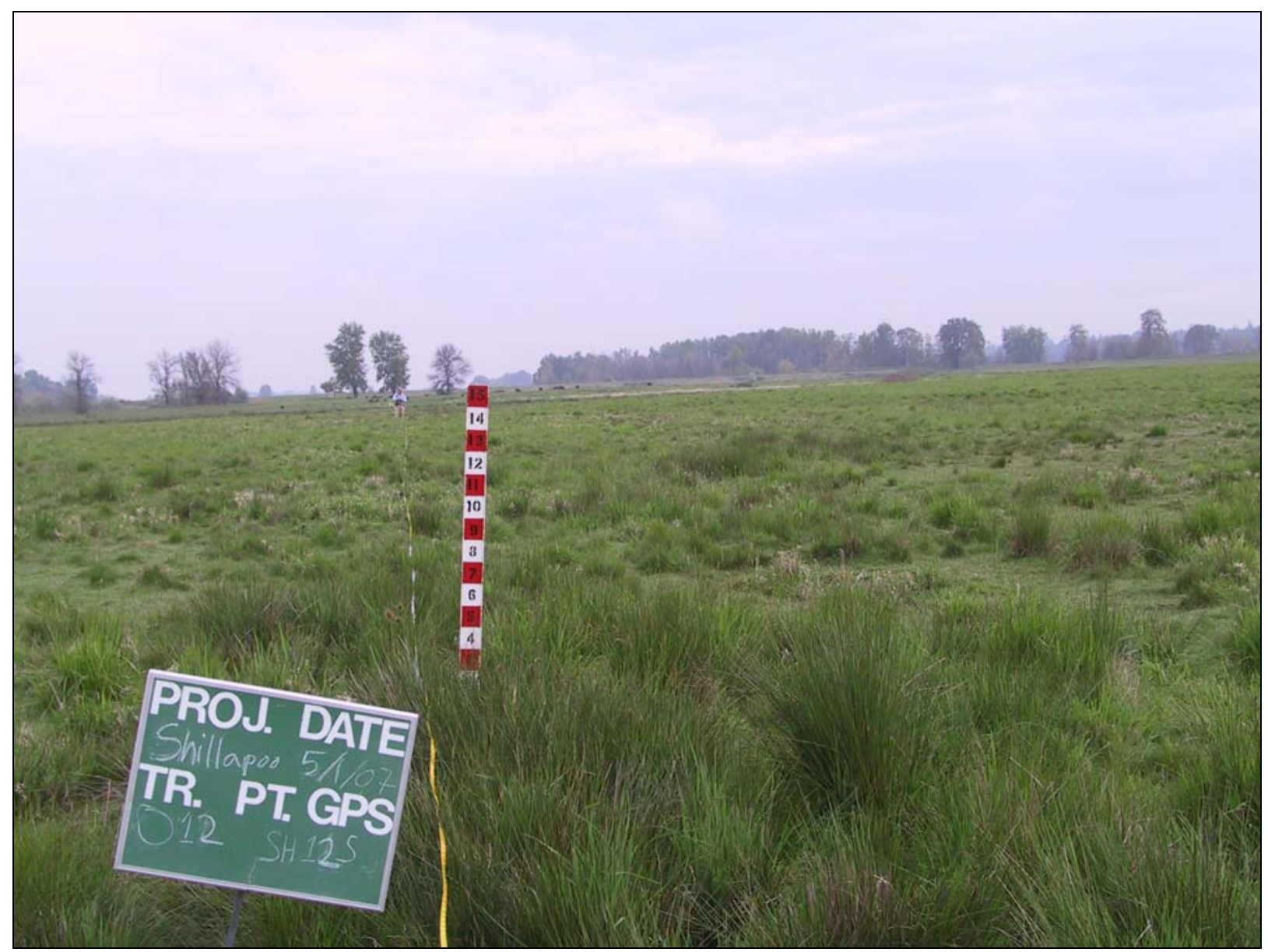

Figure 3. Wet meadow pasture cover type example. 


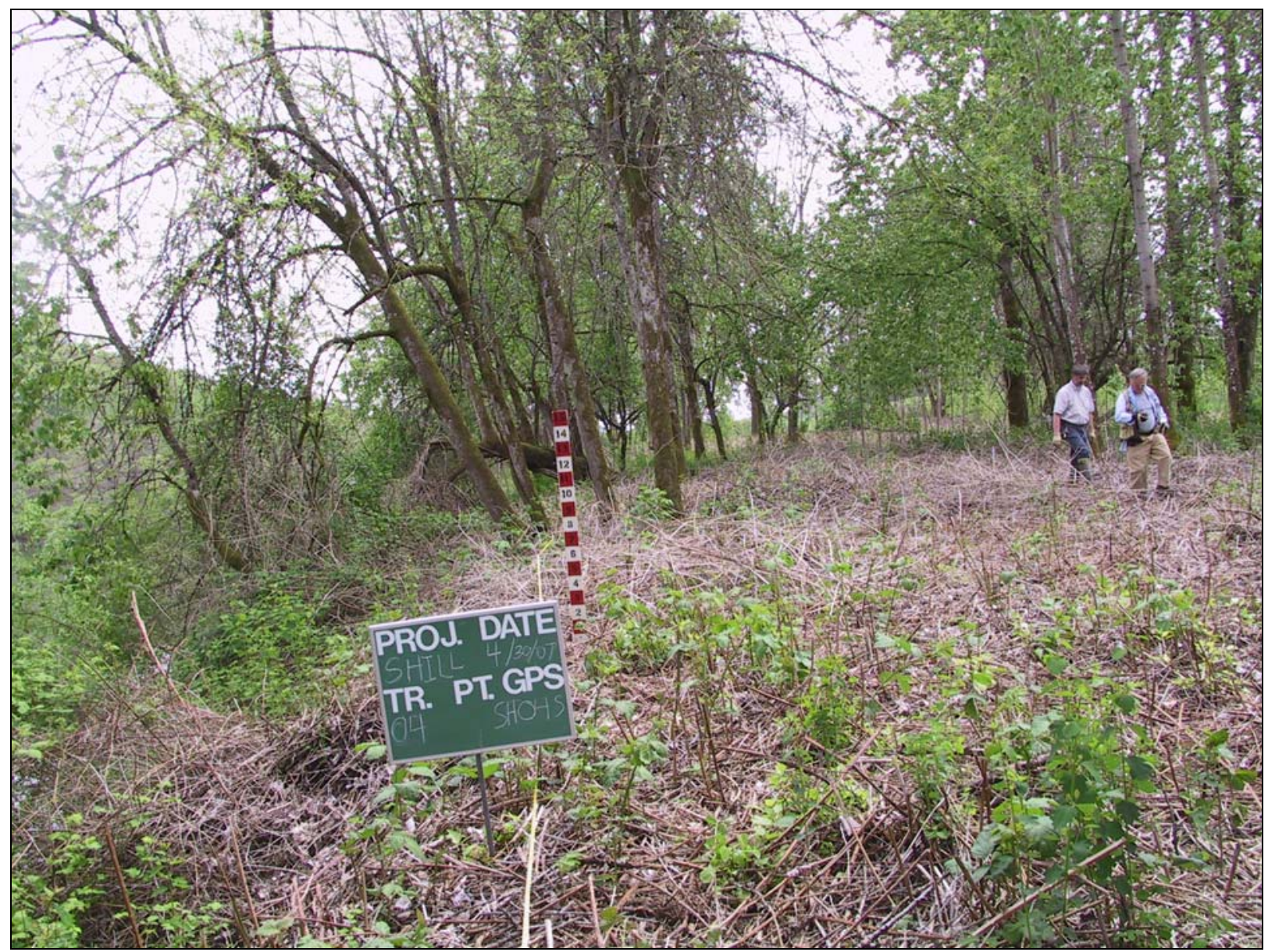

Figure 4. Riparian forest cover type example.

\section{Methods}

\section{Habitat Evaluation Procedures (HEP)}

A habitat evaluation procedures follow-up analysis was conducted on two parcels located at the Shillapoo Wildlife Area to document extant habitat conditions and to determine how many additional habitat units to credit BPA for funding O\&M and enhancement measures. HEP, developed by the U.S. Fish and Wildlife Service (USFWS), is used to quantify the impacts of development, protection, and restoration projects/measures on terrestrial and aquatic habitats by assessing changes, both negative and positive, in habitat quality and quantity (USFWS 1980), (USFWS 1980a).

HEP is a habitat based approach to impact assessment that documents change through use of a habitat suitability index (HSI). The HSI value is derived from an evaluation of the ability of key habitat components to provide the life requisites of selected wildlife and fish species.

The HSI value is an index to habitat carrying capacity for a specific species or guild of species based on a performance measure (e.g. number of deer per square mile) described in HEP species models. The index ranges from 0.0 to 1.0. A HSI of 0.3 indicates that habitat quality/carrying 
capacity is marginal while a HSI of 0.7 suggests that habitat quality/carrying capacity is relatively good for a particular species (Table 2).

Table 2. Habitat suitability index verbal equivalency table.

\section{Habitat Suitability Index}

\begin{tabular}{|c|c|}
\hline $0.0<0.2$ & Poor \\
\hline $0.2<0.4$ & Marginal \\
\hline $0.4<0.6$ & Fair \\
\hline $0.6<0.9$ & Good \\
\hline $0.9<1.0$ & Optimum \\
\hline
\end{tabular}

Each increment of change is identical. For example, a change in HSI from 0.1 to 0.2 represents the same magnitude of change as a change from 0.2 to 0.3 , and so forth. Habitat variables, suggested mensuration techniques, and mathematical aggregations of assessment results are included in HEP evaluation species models.

Habitat units are determined by multiplying the habitat suitability index by the number of acres of habitat (cover type) protected. For example, if the HSI output for a mule deer HEP model is 0.5 and the number of acres of shrubsteppe habitat protected is 100 , then the number of HUs are 50 (0.5 HSI x 100 acres $=50$ HUs).

\section{HEP Model Selection}

HEP models selected by the RHT to assess extant habitat conditions included black-capped chickadee (Parus atricapillus) (Schroeder 1983), great blue heron (Ardea herodias) ${ }^{2}$, Canada goose (Branta canadensis) ${ }^{2}$, mallard (Anas platyrhynchos) ${ }^{2}$, and mink (Neovison vison) ${ }^{2}$. HEP evaluation species selections was based on current cover types and were the same models used to evaluate 1994 baseline habitat conditions ${ }^{2}$. The SWA cover type/species matrix is illustrated in Table 3. Abbreviated HEP models are included in Appendix A.

Table 3. Shillapoo Wildlife Area 2007 follow-up HEP cover type/species matrix.

\begin{tabular}{|l|c|l|l|}
\hline \multicolumn{1}{|c|}{ Cover Type } & Acres & \multicolumn{1}{c|}{ Models } & \multicolumn{1}{c|}{ Credited Dam } \\
\hline Wet Meadow Pasture & 777 & Heron, Canada Goose & Bonneville \\
\hline Emergent Wetland & 40 & Mallard, Mink & John Day \\
\hline Riparian Forest & 5 & Heron, Black-capped Chickadee & Bonneville \\
\hline \multicolumn{1}{r|}{ Total } & $\mathbf{8 2 2}$ & & \\
\hline
\end{tabular}

HEP model selection was based on habitat types and species models identified in the Bonneville Dam and John Day hydro facility loss assessments (Rasmussen and Wright 1989) and were identical to those described by Bich et. al. (1991) for Yakama Nation HEP projects. Loss assessment matrices for Bonneville and John Day Dams are shown in Table 4 and Table 5.

\footnotetext{
${ }^{2}$ HEP models were the same models use by the Yakama Nation to credit BPA for funding mitigation projects (Bich et. al. 1991).
} 
Table 4. Bonneville Dam loss assessment matrix.

\begin{tabular}{|c|c|c|c|c|c|c|c|c|}
\hline \multirow[b]{2}{*}{ HEP MODEL } & \multicolumn{8}{|c|}{ BONNEVILLE DAM COVER TYPE/SPECIES MATRIX } \\
\hline & Rip. Tree & Rip. Shrub & $\begin{array}{l}\text { Wetlands, } \\
\text { Lakes, and } \\
\text { Ponds }\end{array}$ & $\begin{array}{c}\mathrm{Sa} / \mathrm{Gr} \\
\mathrm{Co} / \mathrm{Mud}^{1}\end{array}$ & $\begin{array}{l}\text { Open Water, } \\
\text { Reservoir, }^{\text {River }}\end{array}$ & Islands & $\begin{array}{l}\text { Conifer- } \\
\text { Hardwood } \\
\text { Forest }\end{array}$ & $\begin{array}{l}\text { Shrub- } \\
\text { steppel } \\
\text { Grassland }^{3}\end{array}$ \\
\hline Canada Goose & & & $x$ & $x$ & & $x$ & & $x$ \\
\hline Spotted Sandpiper & & & $x$ & $x$ & & & & \\
\hline Mink & & & $x$ & $x$ & $x$ & & & \\
\hline Black-capped Chickadee & $x$ & & & & & & $x$ & \\
\hline Yellow Warbler & & $x$ & & & & & & \\
\hline Great Blue Heron & $x$ & & $\mathrm{x}$ & $\mathrm{x}$ & $x$ & & & $\mathrm{x}$ \\
\hline TOTAL & 2 & 1 & 4 & 4 & 2 & 1 & 1 & 2 \\
\hline
\end{tabular}

${ }^{1}$ Sand, gravel, cobble, and mud cover type

${ }^{2}$ The open water cover type includes 1,336 scaup HU gains (50\% of 2,671 (HUs). HU gains are not included in this matrix.

${ }^{3}$ Includes wet meadow pasture

Table 5. John Day Dam loss assessment matrix.

\begin{tabular}{|c|c|c|c|c|c|c|c|c|c|}
\hline \multirow[b]{2}{*}{ HEP MODEL } & \multicolumn{9}{|c|}{ JOHN DAY DAM COVER TYPEISPECIES MATRIX } \\
\hline & Rip. Tree & Rip. Shrub & Rip. Herb & $\begin{array}{c}\mathrm{Sa} / \mathrm{Gr} \\
{\mathrm{Co} / \mathrm{Mud}^{1}}^{1}\end{array}$ & $\begin{array}{l}\text { Emergent } \\
\text { Wetland }\end{array}$ & $\begin{array}{c}\text { Shrub- } \\
\text { steppel } \\
\text { Grassland }\end{array}$ & Agricultural & Islands & Open Water ${ }^{2}$ \\
\hline California Quail & & & & & & $x$ & & & \\
\hline Canada Goose & & & $x$ & & & & $x$ & $x$ & \\
\hline Mallard & & & $x$ & & $x$ & & & $x$ & \\
\hline Spotted Sandpiper & & & & $x$ & & & & & \\
\hline Mink & & $x$ & & & $x$ & & & & \\
\hline Western Meadowlark & & & & & & $x$ & & & \\
\hline Black-capped Chickadee & $x$ & & & & & & & & \\
\hline Yellow Warbler & & $x$ & & & & & & & \\
\hline Great Blue Heron & & & & $x$ & & & & & \\
\hline TOTAL & 1 & 2 & 2 & 2 & 2 & 2 & 1 & 2 & 0 \\
\hline
\end{tabular}

${ }^{1}$ Sand, gravel, cobble, and mud cover type.

${ }^{2}$ The open water cover type includes 7,199 scaup $\mathrm{HU}$ gains $(50 \%$ of $14,398 \mathrm{HUs})$. HU gains are not included in this matrix. 


\section{Sampling Design and Measurement Protocols}

\section{Meta Data}

Level one meta data follows that suggested by Gotelli and Ellison (2004). Field surveys were conducted by the Columbia Basin Fish and Wildlife Authority Regional HEP Team with assistance from Shillapoo Wildlife Area staff. Regional HEP Team members included Paul Ashley (RHT Coordinator), Mike Cantonese (Team Leader), Anthony Muse, and Paul Walker, (contact Paul Ashley@ lonepinebutte@comcast.net, or through CBFWA at: [503] 229-0191).

Funding for the HEP analyses was provided by the Bonneville Power Administration with RHT administrative support provided by CBFWA. Specific measurement techniques and protocols are described in detail in Appendix B. Measurements were recorded in standard U.S. units.

\section{Transect Methods}

In most cases, the Regional HEP team used measurement techniques and protocols described in HEP models to evaluate habitat variables; however, ocular estimations were used when direct measurements could not be taken. Measured techniques were occasionally modified to meet unique habitat and/or physiographic conditions. Metrics generally followed those described by Hays et al. (1981) and/or Avery (1994).

Stratified (by cover type), random transects were established and documented using global positioning system (GPS) coordinates (transect rebar stakes were not left in the field). Ashley (2006) described the methods and protocols used by Regional HEP Team staff to collect HEP model variable data and additional floristic information (Appendix B). Field data was summarized and applied to HEP model variables to determine habitat suitability indices and habitat units for each HEP species model. Field data collection and processing procedures are illustrated in Figure 5 and summarized as follows.

HEP model variable field data was entered onto Allegro CE® data logger spreadsheets (1), or recorded on paper data sheets (2). The raw field data (3) was downloaded from the data loggers or manually entered from paper data sheets onto computers (transect photos were also downloaded and stored on field computers). The raw data and photos were compiled for each transect into three basic products/files (4) that are provided to project managers as report appendices and/or separate CD files.

Product files included raw field data downloaded from the data loggers (5), data summary spreadsheets (6) which are the results of compiling/processing the raw data, and transect photo files (7). Summarized/processed data from each transect was applied to appropriate HEP model variables to determine suitability index (SI) ratings that were combined on habitat suitability index (HSI) spreadsheets (8) to determine the HSI for a particular HEP species model/cover type. The habitat suitability index was then multiplied by the number of cover type acres to determine the number of habitat units (9). 


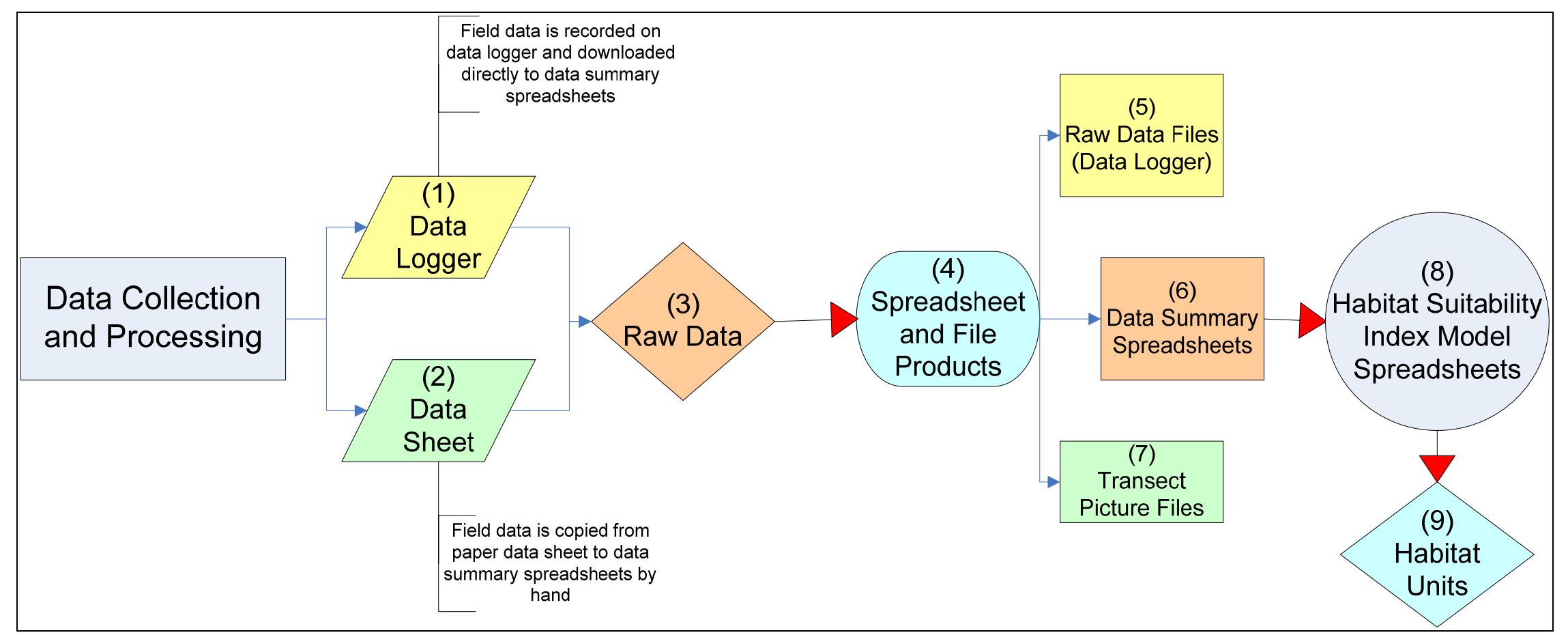

Figure 5. HEP data collection and processing flow chart. 


\section{Transect Locations}

Baseline (1994) transect coordinates were not available for the 2007 follow-up HEP ${ }^{3}$. As a result, baseline transect locations could not be re-evaluated. New transect locations were established as follows.

Transect initial points (IPs) were established based on stratified random sampling protocols with cover types defining the strata. The number of samples initially allocated per cover type strata were determined based on a proportional allocation strategy (Husch et al. 2003). Specific IP locations were identified by overlaying a $100 \mathrm{~m} \times 100 \mathrm{~m}$ grid over cover types and selecting random numbers to identify " $\mathrm{XY}$ " point coordinates ( $\mathrm{P}$. Ashley, pers. comm.)

The proportional allocation strategy was modified in the field as needed to compensate for the relative homogeneity of a particular cover type, to account for unanticipated access issues and/or physiographic restrictions, and/or to meet temporal considerations. In addition, initial points were moved when they did not fall within the cover type(s) of interest, or located in inaccessible areas such as the middle of a pond or cliff area (additional transect information is located in Appendix B).

Transect UTM coordinates (NAD 27) for start and turn points were recorded in the field on a Garmin IIIA ${ }^{\circledR}$ GPS unit. Transect start $(\mathrm{S})$ and end $(\mathrm{E})$ points are illustrated in Figure 6 and Figure 7. IP/transect UTM coordinates, transect magnetic azimuths, and transect lengths are summarized in Table 6.

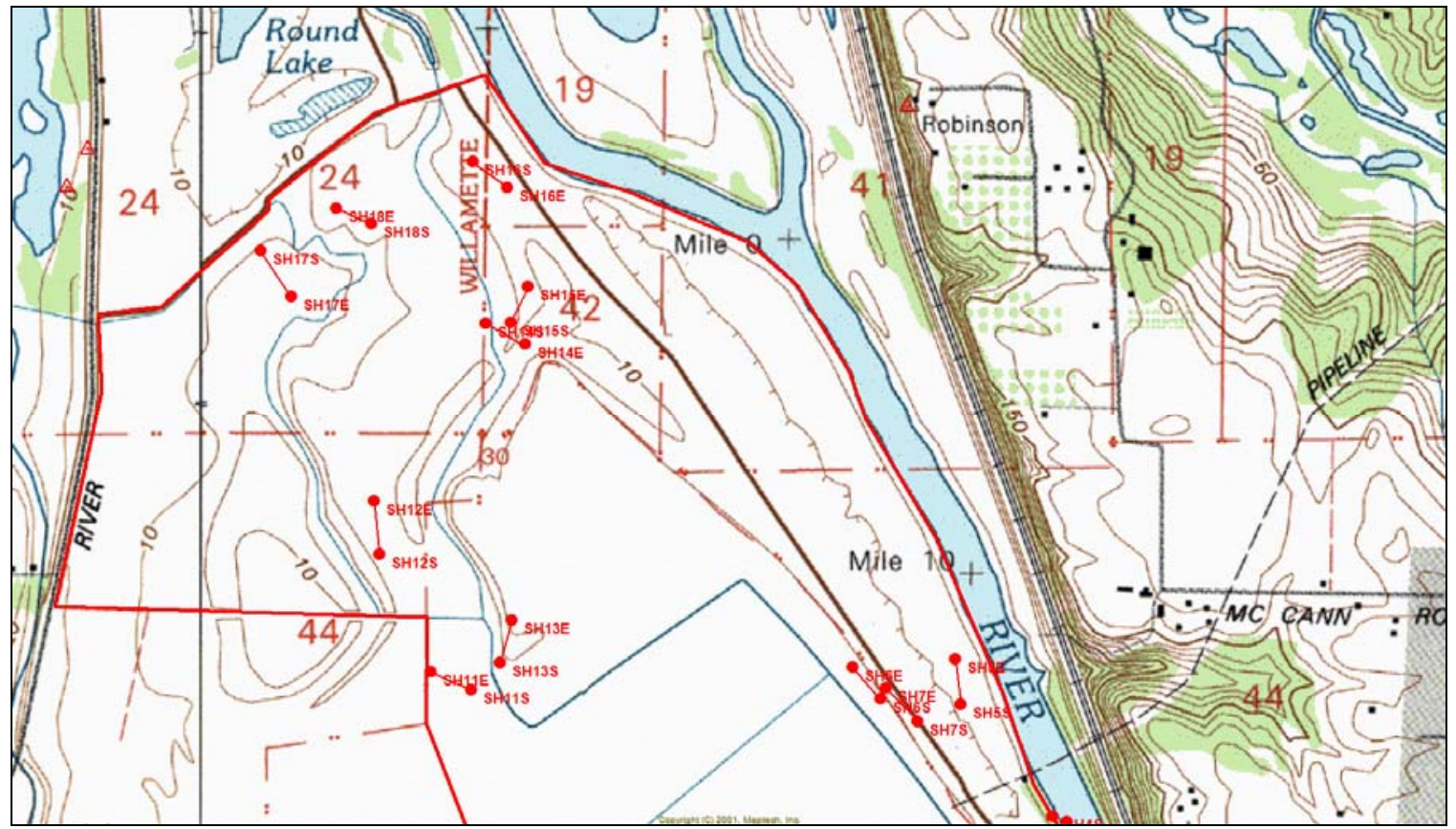

Figure 6. Follow-up HEP transect location start (S) and end (E) points (north half of SWA).

${ }^{3}$ HEP survey staff did not possess GPS equipment in 1994. 
Shillapoo WA 2007 HEP Report

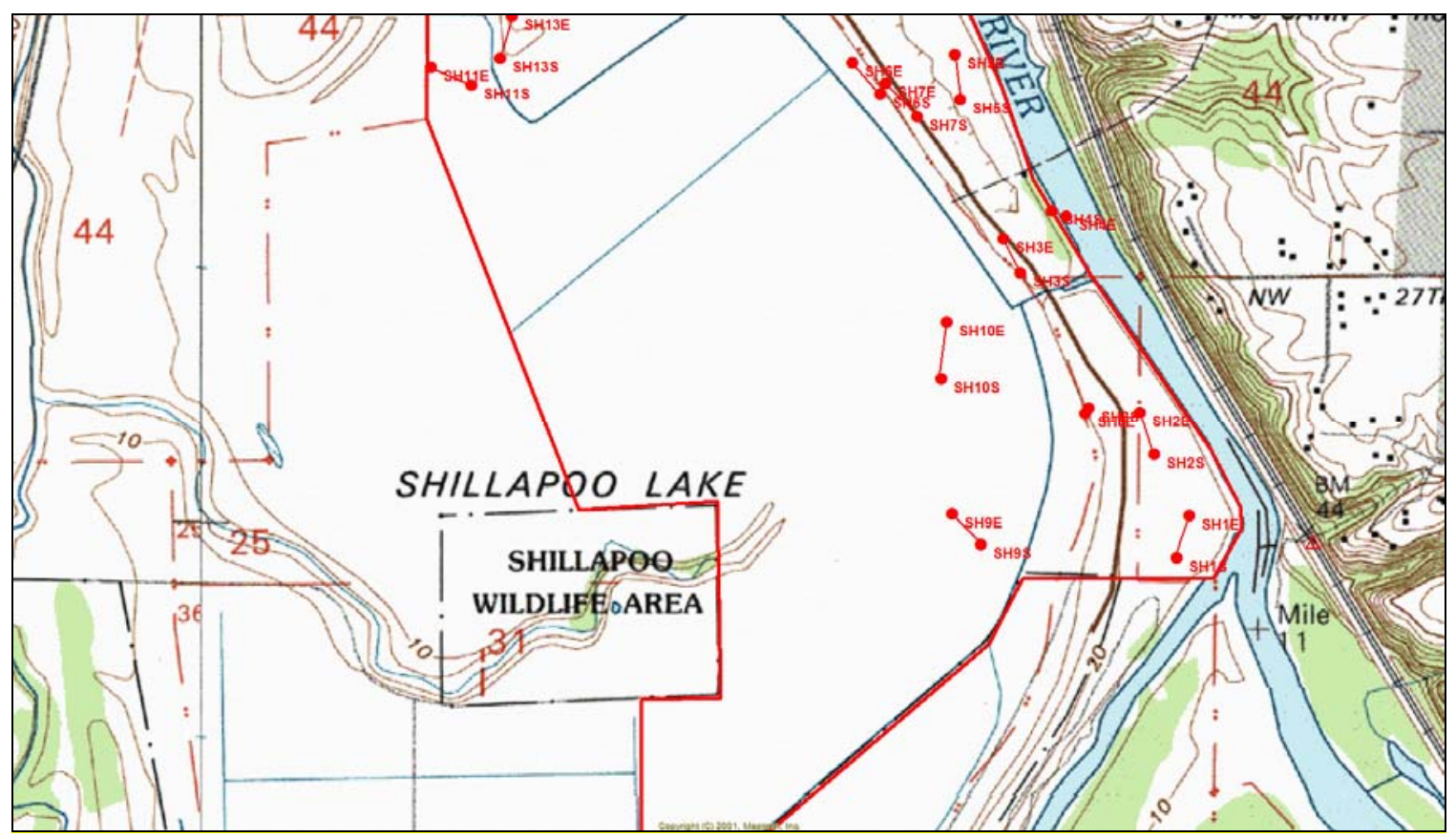

Figure 7. Follow-up HEP transect location start (S) and end (E) points (south half of SWA).

Table 6. Shillapoo Wildlife Area 2007 follow-up HEP transect coordinates, azimuths, and lengths.

\begin{tabular}{|c|c|c|c|c|c|c|}
\hline \multirow{2}{*}{ Project \& Transect No. } & \multirow{2}{*}{ Point } & \multicolumn{2}{|c|}{ GPS } & \multirow{2}{*}{$\begin{array}{l}\text { Magnetic } \\
\text { Azimuth } \\
\text { (Degrees) }\end{array}$} & \multirow{2}{*}{$\begin{array}{l}\text { Length } \\
\text { (Feet) }\end{array}$} & \multirow{2}{*}{ Total Length } \\
\hline & & $E$ & $\mathbf{N}$ & & & \\
\hline WDFW-Shillapoo & & $10 U$ & & & & \\
\hline \multirow[t]{2}{*}{1} & start & 0521540 & 5061386 & 009 & 300 & 300 \\
\hline & end & 0521565 & 5061476 & & & \\
\hline \multirow[t]{2}{*}{2} & start & 0521491 & 5061607 & 326 & 300 & 300 \\
\hline & end & 0521459 & 5061696 & & & \\
\hline \multirow[t]{2}{*}{3} & start & 0521205 & 5061994 & 309 & 300 & 300 \\
\hline & end & 0521168 & 5062067 & & & \\
\hline \multirow[t]{2}{*}{4} & start & 0521271 & 5062127 & 140 & 300 & 300 \\
\hline & end & 0521301 & 5062115 & & & \\
\hline \multirow[t]{2}{*}{5} & start & 0521076 & 5062363 & 325 & 300 & 300 \\
\hline & end & 0521063 & 5062459 & & & \\
\hline \multirow[t]{2}{*}{6} & start & 0520905 & 5062374 & 333 & 300 & 300 \\
\hline & end & 0520846 & 5062442 & & & \\
\hline \multirow[t]{2}{*}{7} & start & 0520984 & 5062346 & 288 & 300 & 300 \\
\hline & end & 0520917 & 5062398 & & & \\
\hline \multirow[t]{2}{*}{8} & start & 0521350 & 5061705 & 191 & 300 & 300 \\
\hline & end & 0521343 & 5061694 & & & \\
\hline \multirow[t]{2}{*}{9} & start & 0521122 & 5061413 & 282 & 300 & 300 \\
\hline & end & 0521049 & 5061475 & & & \\
\hline \multirow[t]{2}{*}{10} & start & 0521038 & 5061768 & 339 & 300 & 300 \\
\hline & end & 0521049 & 5061888 & & & \\
\hline 11 & start & 0520034 & 5062390 & 281 & 300 & 300 \\
\hline
\end{tabular}


Shillapoo WA 2007 HEP Report

\begin{tabular}{|c|c|c|c|c|c|c|}
\hline \multirow{2}{*}{ Project \& Transect No. } & \multirow{2}{*}{ Point } & \multicolumn{2}{|c|}{ GPS } & \multirow{2}{*}{$\begin{array}{l}\text { Magnetic } \\
\text { Azimuth } \\
\text { (Degrees) }\end{array}$} & \multirow{2}{*}{$\begin{array}{l}\text { Length } \\
\text { (Feet) }\end{array}$} & \multirow{2}{*}{ Total Length } \\
\hline & & E & $\mathbf{N}$ & & & \\
\hline & end & 0519946 & 5062411 & & & \\
\hline \multirow[t]{2}{*}{12} & start & 0519839 & 5062677 & 337 & 300 & 300 \\
\hline & end & 0519827 & 5062790 & & & \\
\hline \multirow[t]{2}{*}{13} & start & 0520096 & 5062448 & 351 & 300 & 300 \\
\hline & end & 0520120 & 5062539 & & & \\
\hline \multirow[t]{2}{*}{14} & start & 0520063 & 5063172 & 102 & 300 & 300 \\
\hline & end & 0520145 & 5063127 & & & \\
\hline \multirow[t]{2}{*}{15} & start & 0520117 & 5063175 & 003 & 300 & 300 \\
\hline & end & 0520153 & 5063251 & & & \\
\hline \multirow[t]{2}{*}{16} & start & 0520035 & 5063518 & 102 & 300 & 300 \\
\hline & end & 0520107 & 5063461 & & & \\
\hline \multirow[t]{2}{*}{17} & start & 0519584 & 5063326 & 124 & 300 & 300 \\
\hline & end & 0519651 & 5063228 & & & \\
\hline \multirow[t]{2}{*}{18} & start & 0519820 & 5063358 & 296 & 300 & 300 \\
\hline & end & 0519744 & 5063417 & & & \\
\hline
\end{tabular}

\section{Transect Photo Documentation}

Transects were photographed with a Canon G1® 3.3 mega pixal digital camera (with and without magnification). Transect photographs are included in Appendix C.

\section{Photo Methods}

Photo points were established at the start point of each transect to document extant habitat conditions. Digital photographs were recorded from a height of three feet $(\approx 1$ meter) at the beginning of each transect facing the same direction as the transect azimuth. A transect reference board ${ }^{4}$ was placed at the 15 foot interval while a cover board, divided into 3 inch $\times 4$ inch $(8 \mathrm{~cm} \times 10 \mathrm{~cm})$ rectangles, was set at the 30 foot mark on each transect. Panoramic photographs were also recorded to document dense vegetation, linear/narrow cover types, etc. An example of a photo documentation point is illustrated in Figure 8.

\footnotetext{
${ }^{4}$ Showing transect number, project name, date, GPS reference number
} 


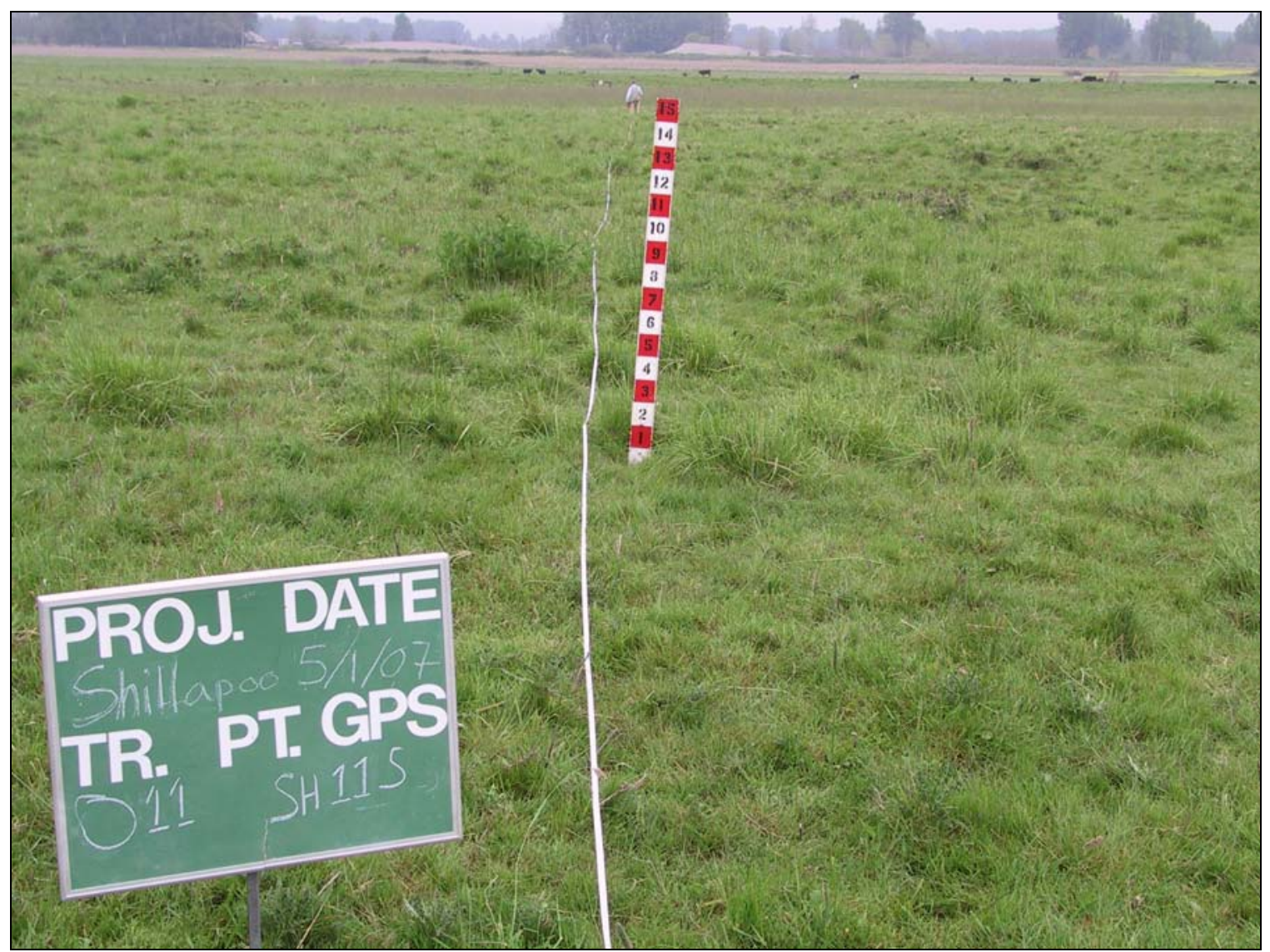

Figure 8. Photo point example.

\section{Results}

The follow-up Habitat Evaluation Procedures evaluation was conducted on the Egger and Herzog parcels (Shillapoo Wildlife Area) in late April/early May 2007. Slightly more than 936 habitat units (936.47) or 1.14 HUs per acre were generated. Results included 1.65 black-capped chickadee HUs, 280.57 great blue heron HUs, 581.45 Canada goose HUs, 40 mallard HUs, and 32.80 mink HUs.

HEP survey results are summarized by cover types and species in Table 7. HEP species models and habitat suitability mathematical aggregations are included in Appendix A. 
Table 7. Shillapoo Wildlife Area 2007 HEP results summary.

\begin{tabular}{|c|c|c|c|c|c|c|}
\hline Cover Type $^{1}$ & Acres $^{2}$ & Model & Variable & sı & HSI & HUs \\
\hline \multirow{9}{*}{ Riparian Forest } & \multirow{9}{*}{5} & \multirow{3}{*}{$\begin{array}{l}\text { Black-capped } \\
\text { Chickadee }\end{array}$} & V1: Percent Canopy Closure & 0.98 & $0 . .33$ & 1.65 \\
\hline & & & V2: Average Height of Trees & 1.00 & & \\
\hline & & & V4: Number of Snags $10-25 \mathrm{~cm}$ dbh/0.4 ha (6-10"/acre) & 0.33 & & \\
\hline & & \multirow{6}{*}{ Great Blue Heron } & V1: Distance between Foraging Areas and Nesting Sites $(\mathrm{km})$ & 0.90 & 0.17 & 0.85 \\
\hline & & & V2: Foraging Hasbitat Quality & 0.50 & & \\
\hline & & & V3: Human Disturbance Level Near Potential Foraging Zone & 0.80 & & \\
\hline & & & V4: Availability of Potential Nesting Areas & 0.10 & & \\
\hline & & & V5: Disturbance Level in Vicinity of Potential Nesting Areas & 1.00 & & \\
\hline & & & V6: Distance Between Potential Nest Site and Active Site & 0.80 & & \\
\hline Total & 5 & & & & & 2.50 \\
\hline \multirow{7}{*}{ Wet Meadow/Pasture } & \multirow{7}{*}{777} & \multirow{3}{*}{ Great Blue Heron } & V1: Distance between Foraging Areas and Nesting Sites $(\mathrm{km})$ & 0.90 & 0.36 & 279.72 \\
\hline & & & V2: Foraging Hasbitat Quality & 0.50 & & \\
\hline & & & V3: Human Disturbance Level Near Potential Foraging Zone & 0.80 & & \\
\hline & & & & & & \\
\hline & & \multirow{3}{*}{ Canada Goose } & V1: Tree structure & 0.80 & 0.75 & 581.45 \\
\hline & & & V2: Brood Areas & 0.80 & & \\
\hline & & & V3: Human Disturbance & 0.60 & & \\
\hline Total & 777 & & & & & 861.17 \\
\hline \multirow{4}{*}{ Emergent Wetland (ocular) } & \multirow{4}{*}{40} & Mallard & V7: Ratio of Vegetative Cover and Open Water & 1.00 & 1.00 & 40.00 \\
\hline & & \multirow{3}{*}{ Mink } & V1: Percent of year with Surface Water present & 1.00 & .82 & 32.80 \\
\hline & & & V4: Percent Canopy Cover of Emergent Vegetation & 1.00 & & \\
\hline & & & $\begin{array}{l}\text { V5: Percent of tree/shrub canopy closure within 100m (328ft) } \\
\text { of water's or wetland's edge }\end{array}$ & 0.10 & & \\
\hline Total & 40 & & & & & 72.80 \\
\hline Project Total & 822 & & & & & 936.47 \\
\hline \multicolumn{7}{|c|}{$\begin{array}{l}1 \text { The Shillapoo WA is transitioning from active agriculture to wet meadow and emergent wetland cover types. The wet meadow cover type replaced the active } \\
\text { agriculture cover type in 2007. Additional emergent wetlands are planned and will likely be present prior to the next follow-up HEP analysis (B. Calkins, WDFW, pers. } \\
\text { comm. November 2007). }\end{array}$} \\
\hline
\end{tabular}




\section{Discussion (HSI)}

Because cover types and associated acreages were significantly different in 2007 when compared to conditions during the $1994^{5}$ baseline HEP surveys, a direct comparison between HU results cannot be made. On the other hand, habitat suitability indices for like cover types/species are comparable and are depicted in Table 8. HEP results for individual cover types are discussed below.

Table 8. Follow-up and baseline habitat suitability index comparison summary.

\begin{tabular}{|c|c|c|c|c|}
\hline \multirow{2}{*}{ Cover Type } & \multirow{2}{*}{ Model } & \multicolumn{2}{|c|}{ Year/HSI } & \multirow{2}{*}{$\begin{array}{c}\text { Change } \\
\text { in } \mathrm{HSI}\end{array}$} \\
\hline & & 2007 & 1994 & \\
\hline \multirow{3}{*}{ Riparian Forest } & Black-capped Chickadee & 0.33 & 1.00 & -0.67 \\
\hline & & & & \\
\hline & Great Blue Heron & 0.17 & 0.00 & +0.17 \\
\hline \multirow{3}{*}{ Wet Meadow Pasture } & Great Blue Heron & 0.36 & 0.40 & -0.04 \\
\hline & & & & \\
\hline & Canada Goose & 0.75 & 0.72 & +0.03 \\
\hline \multirow{3}{*}{ Emergent Wetland } & Mallard & 1.00 & 0.79 & +0.21 \\
\hline & & & & \\
\hline & Mink & 0.82 & 0.49 & +0.33 \\
\hline
\end{tabular}

\section{Riparian Forest}

\section{Black-capped Chickadee}

The lower 2007 follow-up HEP habitat suitability index (0.33) is the result of "pooling" 2007 HEP data from multiple transects and the lack of snags at two out of three surveyed sites. The lack of suitable snags is the most limiting factor.

\section{Great Blue Heron}

The lack of suitable nesting sites (trees) on project lands is currently the most limiting factor. Heron habitat suitability will remain marginal until riparian forest structural conditions improve.

\section{Wet Meadow Pasture}

\section{Great Blue Heron}

Follow-up HEP survey results indicate that habitat suitability decreased slightly relative to 1994 baseline HEP results. The lower HSI value in 2007 is insignificant and is likely an artifact of different individuals evaluating subjective model habitat variables. The

\footnotetext{
${ }^{5}$ HEP survey year (1994) was provided by Brian Calkins-Shillapoo Wildlife Area Manager.
} 
most significant limiting factor appeared to be forage habitat quality (V2). The Regional HEP Team estimated that dense herbaceous cover and limited shallow surface water reduced prey abundance and/or availability.

\section{Canada Goose}

Like heron HSI results, the difference between 2007 and 1994 habitat suitability is negligible. The disparity is likely an artifact of different individuals evaluating model habitat variables.

\section{Emergent Wetland}

\section{Mallard}

Mallard habitat suitability improved significantly over baseline habitat conditions due to ideal open water to emergent cover ratios at the evaluated site. Mallard brood rearing habitat quality is "optimum" (1.0) based on model output.

\section{Mink}

Mink habitat suitability also improved significantly compared to 1994 baseline HEP results. Although woody cover is limited within 100 meters of the wetland (V4 $=0.10)$, mink model output emphasizes the amount of emergent vegetation present (V3), which compensates somewhat for the lack of adjacent tree and shrub cover.

\section{Epilogue}

This part of the Shillapoo wildlife area will change significantly within the next five years as WDFW implements planned wetland enhancements and other habitat improvement measures. As a result, subsequent HEP evaluation cover types and acreages may be considerably different than found at either 2007 follow-up or 1994 baseline HEP surveys.

\section{Acknowledgements}

I gratefully acknowledge the hard work and effort provided by WDFW Shillapoo Wildlife Area Manager Brian Calkins and Regional HEP Team members Mikael Cantonese, Tiffany Baker, Tony Muse, and Paul Walker. I also want to thank John Talmadge (WDFW) for providing GIS support and Dan Budd (WDFW Real Estate Division) for responding to a myriad of acquisition related questions in a timely manner. 


\section{References}

Ashley, P. R. 2006. Habitat evaluation procedures standard measurement protocols and techniques (draft). Columbia Basin Fish and Wildlife Authority (CBFWA). Portland, OR.

Avery, T.E., H. E. Burkhart. 1994. Forest measurements. $4^{\text {th }}$ edition. New York, NY: John Wiley and Sons.

Bich, J. P., T. Hames, S. McCorquodale, J. D. Reichel, and W. P. Bradley. 1991. The Yakima Indian Nation wildlife mitigation plan for Bonneville, The Dalles, John Day, and McNary Dams. Draft report for public comment. Yakima Indian Nation Wildlife Resource Management. Toppenish, WA.

BPA and WDFW. 1996. Memorandum of Agreement between the Washington Department of Fish and Wildlife and Bonneville Power Administration for the disbursal of wildlife mitigation funds and mitigation crediting. WDFW. Olympia, WA. BPA. Portland, OR.

Gotelli, N. J., A. M. Ellison. 2004. A primer of ecological statistics. Sinauer Associates, Inc. Sunderland, MA.

Hays, R. L., C. Summers, and W. Seitz. 1981. Estimating habitat variables. Western Energy and land Use Team. Fort Collins, CO: U.S. Fish and Wildlife Service.

Husch, B., T.W. Beers, and J.A. Kershaw, Jr. 2003. Forest mensuration- $4^{\text {th }}$ edition. Hoboken, NJ: Wiley and Sons, Inc.

Rasmussen, L., P. Wright. 1989. Wildlife impact assessment Bonneville, McNary, The Dalles, and John Day Projects. Bonneville Project, Oregon and Washington. Annual Report 1989. USDOE. BPA. Portland, Oregon.

Schroeder, R.L. 1983. Habitat suitability index models: Black-capped Chickadee. U.S. Department of the Interior, Fish and Wildlife Service. FWS/OBS-82/10.37.

USFWS. 1980. Habitat as a Basis for Environmental Assessment, Ecological Services Manual (ESM) 101. Division of Ecological Services, U. S. Fish and Wildlife Service, Washington, DC: Department of the Interior.

- 1980a. Habitat Evaluation Procedures (HEP), Ecological Services Manual (ESM) 102. Division of Ecological Services, U.S. Fish and Wildlife Service, Washington, DC: Department of the Interior. 


\section{Appendix A - Abbreviated HEP Models}

\section{Mallard}

Species:

Model:

Cover Type:

MALLARD

Rasmussen and Wright, 1990b,d

Emergent Wetland, Agricultural, SS

Grassland, Riparian Herb, Riverine, Lacustrine.

Variable 3: Distance between nest \& water with emergent cover (miles)

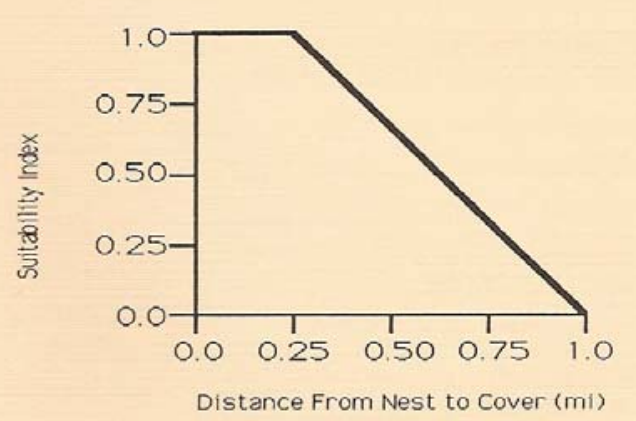

V3 Field Values

$\begin{array}{lll}<0.25 \mathrm{mi} & = & 1.0 \\ 0.25-0.75 \mathrm{mi} & = & 0.5 \\ >0.75 \mathrm{mi} & = & 0.1\end{array}$

Variable 4: Height of residual nesting cover (inches)

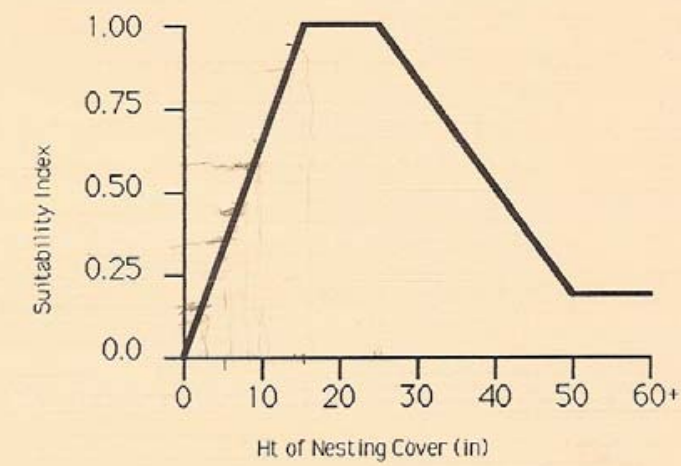

$\underline{\text { V4 Field Values }}$

$\begin{array}{lll}0 \text { in } & = & 0.0 \\ 1-15 \text { in } & = & 0.5 \\ 16-24 \text { in } & = & 1.0 \\ 25-48 \text { in } & = & 0.6 \\ >48 \text { in } & = & 0.3\end{array}$

Variable 5: \% Canopy cover of nesting vegetation

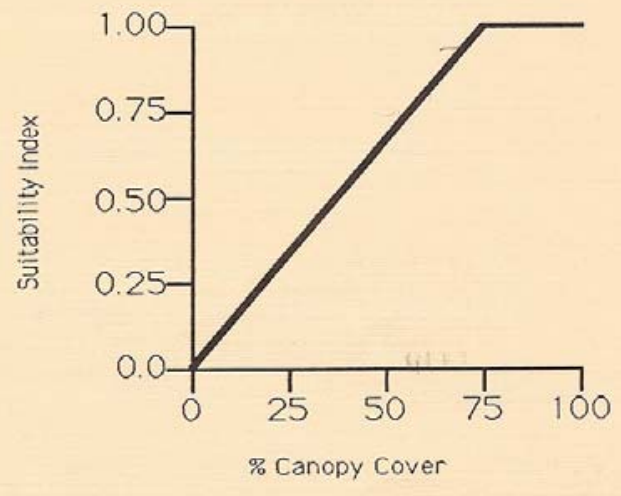

$\underline{\text { V5 Field Values }}$

$\begin{array}{lll}<50 \% & = & 0.3 \\ 51-75 \% & = & 0.7 \\ >75 & = & 1.0\end{array}$


Shillapoo WA 2007 HEP Report

Variable 6: Human disturbance

$\begin{array}{lll}\text { None } & = & 0.8-1.0 \\ \text { Moderate } & = & 0.4-0.7 \\ \text { High } & = & 0-0.3\end{array}$

\section{V6Field Values}

$\begin{array}{lll}\text { None } & = & 1.0 \\ \text { Moderate } & = & 0.5 \\ \text { High } & = & 0.2\end{array}$

Notes: All variables were estimated at the field sampling sites using the field scales.

The mallard model was applied in the field considering estimated vegetative conditions on April 1, the approximate date of mallard nest initiation.

Human disturbance included any disturbance associated with human presence, such as livestock, pets, machinery, and traffic.

Variable 7: Ratio of vegetative cover to open water

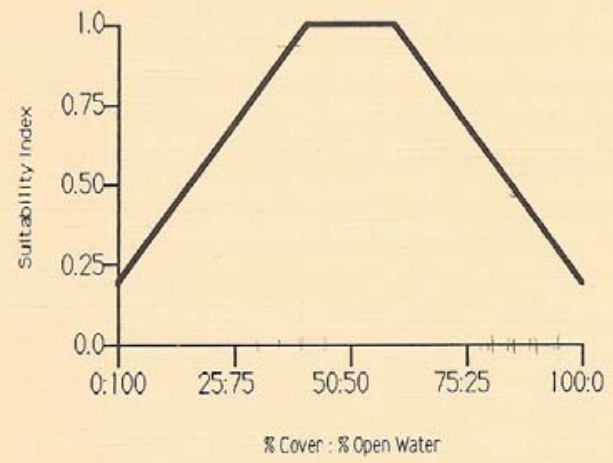

\section{V7 Field Values}

$\begin{array}{lll}<40: 60 & = & 0.5 \\ 40: 60-60: 40 & = & 1.0 \\ >60: 40 & = & 0.5\end{array}$

In emergent wetlands:

Mallard HSI $=$ V7

- In other cover types:

Mallard HSI $=(\mathrm{V} 3+\mathrm{V} 4+\mathrm{V} 5) / 3$ X V6 


\title{
Canada Goose
}

\author{
Species: $\quad$ CANADA GOOSE \\ Model: $\quad$ De Waard 1990 \\ Cover type: Sand/Gravel/Cobble/Mud, Agricultural, SS Grassland, Riparian Herb, \\ Lacustrine.
Variable 1: Mature riparian forest adjacent to river, snags, etc. $=1.0$ Mature trees in limited supply, few snags $\quad=\quad 0.5$ \\ Few mature trees $\quad=0.2$ \\ Variable 3: Brood areas \\ Short grass, easy access $<1$ mile from nesting $\quad=\quad 1.0$ \\ Short grass access restricted or $1-2$ miles from nesting $=0.5$ \\ Brood areas not apparent or $>2$ miles from nesting areas $=0.2$ \\ Variable 4: Human disturbance $>1 / 2$ mile away $\quad=\quad 1.0$ \\ Human disturbance $1 / 4-1 / 2$ mile away $=0.5$ \\ Human disturbance $<1 / 4$ mile away $\quad=0.1$ \\ Canada Goose HSI $=[\mathrm{V} 1 \times(\mathrm{V} 3+\mathrm{V} 4) / 2]^{1 / 2}$
}

Notes: Nesting goose HUs lost through inundation by the Lower Columbia River Project were primarily associated with the mainstem Columbia island cover type. Due to the breadth of the Columbia channel and the distance from main shoreline to island shorelines, these islands offered isolation from nest predators. The size of the Columbia is unique within the Northwest; along the Yakima River, as well as most other regional streams, islands do not provide the same isolation from predators as was typical of the Columbia. Smaller islands in most regional streams also make them more prone to flooding during spring runoff, substantially reducing their value to ground nesting birds. Therefore, other local cover types provide the bulk of nesting goose habitat along the smaller order streams. Along the Yakima River, riparian forest communities provide the best, most secure habitat for nesting Canada geese. To reflect this, the goose model was modified to provide estimates of the HUs available for nesting geese in the local project area. Canada geese were selected in the loss assessment due to their regional significance, not due to the importance of islands per se.

Human disturbance was considered any disturbance associated with human presence. These disturbances included livestock, pets, machinery, traffic, etc. 


\section{Great Blue Heron}

Species: $\quad$ GREAT BLUE HERON

Model: $\quad$ Short and Cooper 1985.

Cover Type: Riparian Forest, Sand/Gravel/Cobble/Mud, SS Grassland, Riverine, Lacustrine.

Variable 1: Distance from feeding area to potential nesting area $(\mathrm{km})$

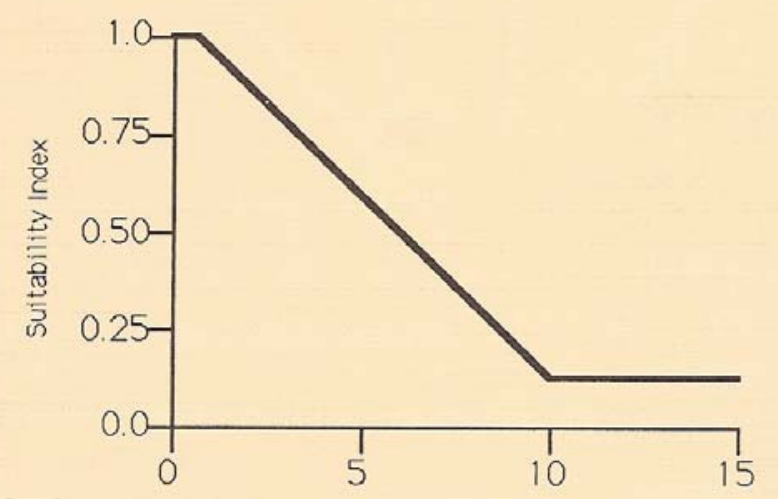

Distance Between Foraging and Potential Nesting Areas (km)

Variable 2: Foraging habitat quality

Shallow, clear water with firm substrate and forage fish $=1.0$

Wet pasture (see notes below) $\quad=0.5$

Quality foraging conditions absent or scarce $=0.0$

Variable 3: Human disturbance level near potential foraging zone

No frequent human disturbance within $100 \mathrm{~m}$

$=\quad 1.0$

( or foraging zone $\geq 50 \mathrm{~m}$ from low-use road)

Frequent disturbance within $100 \mathrm{~m}$

$=0.0$

Variable 4: Availability of potential nesting areas

Trees $\geq 5 \mathrm{~m}$ with an open canopy located $\leq 250 \mathrm{~m}$ from water $=\quad 1.0$

Quality nesting conditions absent or scarce

$=\quad 0.0$

Variable 5: Disturbance level in vicinity of potential nesting areas

Low disturbance within $250 \mathrm{~m}$ on land or $150 \mathrm{~m}$ on water $=\quad 1.0$

Disturbance sources within $250 \mathrm{~m}$ on land or $150 \mathrm{~m}$ on water $=\quad 0.0$ 
Shillapoo WA 2007 HEP Report

Variable 6: Distance between potential nest site and nearest active nest site

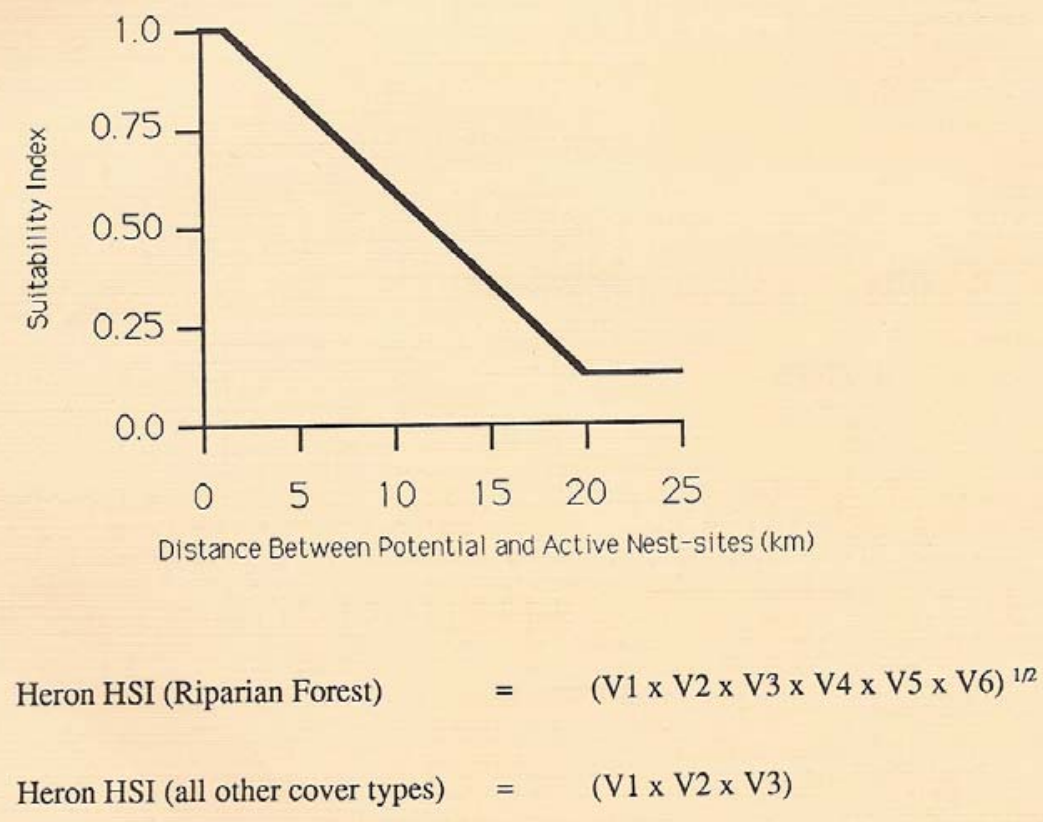

Notes: All variables estimated from 1:20,000 color aerial photographs. The continuous variable function was used for variable 1 . Modification of variable 2 reflected some foraging value associated with wet pastures. Observations indicated herons foraged locally in wet pastures, apparently consuming small mammals, snakes, frogs, and possibly some invertebrates.

Human disturbance was considered any disturbance associated with human presence. These disturbances included livestock, pets, machinery, traffic, etc. 


\section{Black-capped Chickadee}

Model Relationships

Suitability Index (SI) graphs for habitat variables. This section contains SI graphs that illustrate the habitat relationships described in the previous section.
Cover
type
$\mathrm{DF}, \mathrm{EF}$,
$\mathrm{DFW}, \mathrm{EFW}$
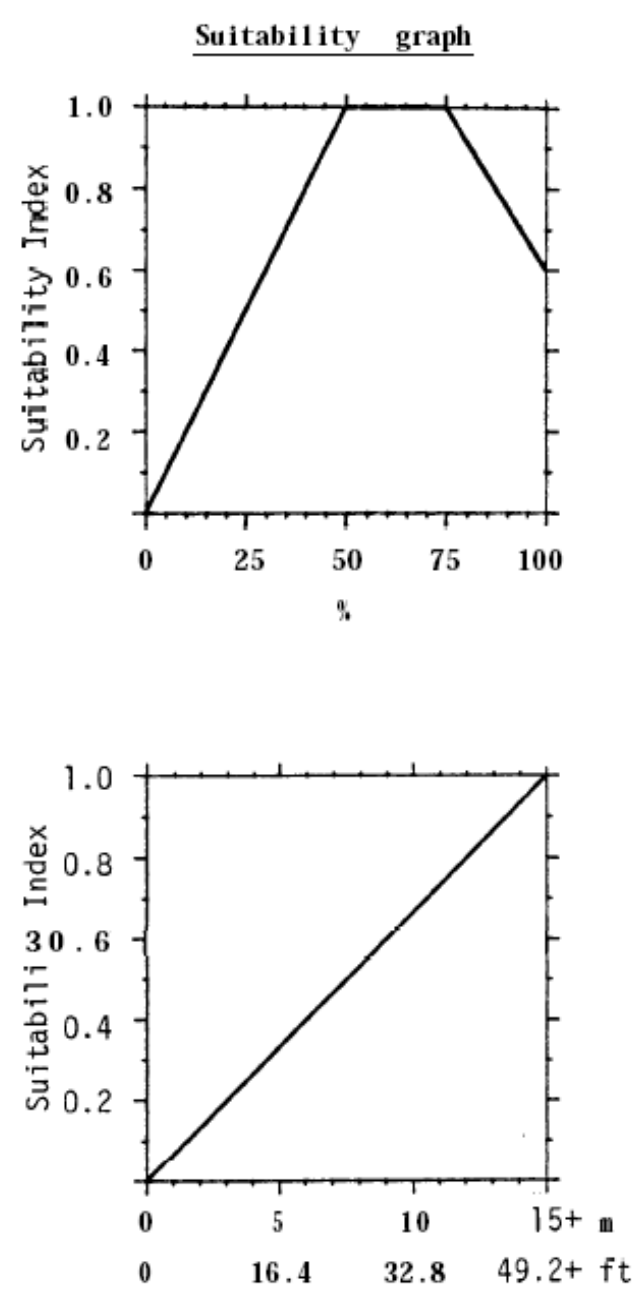
DF, EF,

DFW, EFW
$V_{2}$

Tree canopy volume/ area of ground surface.
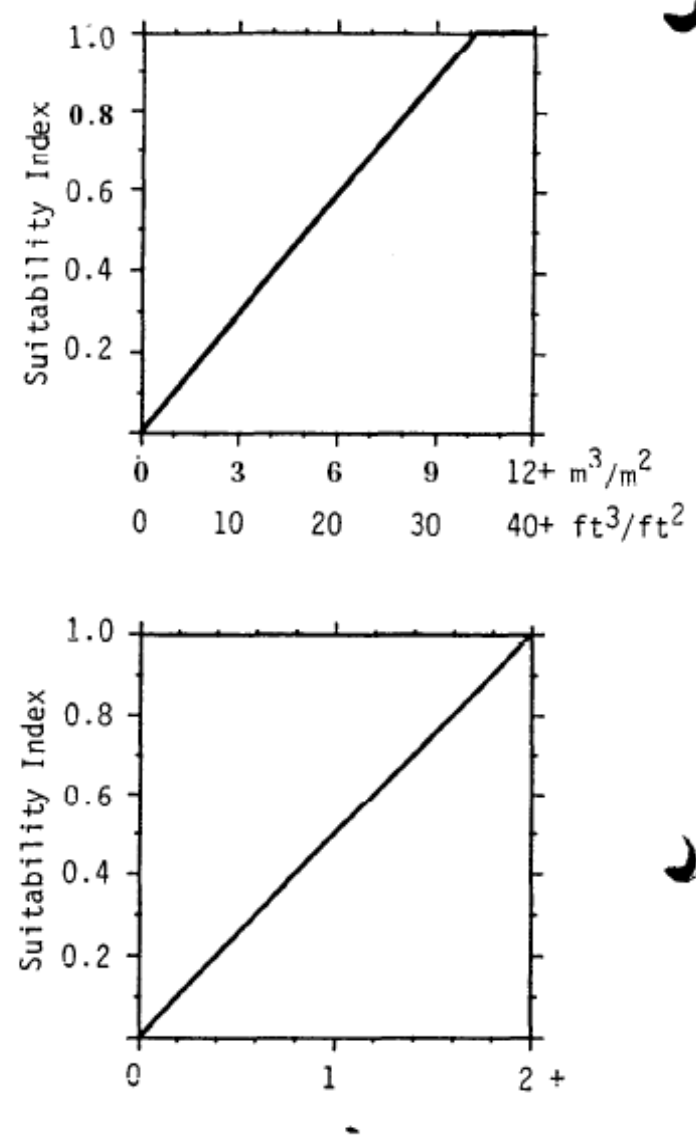

Equation

$\left(V_{1} \times V_{2}\right)^{1 / 2}$ or $V_{3}$ (See page 5 for discussion on which to use)

$\mathrm{V}_{4}$

DF , EF, DFW , EFW

Reproduction

HSI determination. The HSI for the black-capped chickadee is equal to the lowest life requisite value. 
Shillapoo WA 2007 HEP Report

\section{Mink}

$\begin{array}{ll}\text { Species: } & \text { MINK } \\ \text { Model: } & \text { Allen } 1986 \\ \text { Cover Type: } & \text { Riverine, Emergent Wetlands, } \\ & \begin{array}{l}\text { Riparian Forest, Riparian Shrub, } \\ \text { Sand/Gravel/Cobble/Mud }\end{array} \\ \end{array}$

Variable 1: $\%$ of year w/surface water present

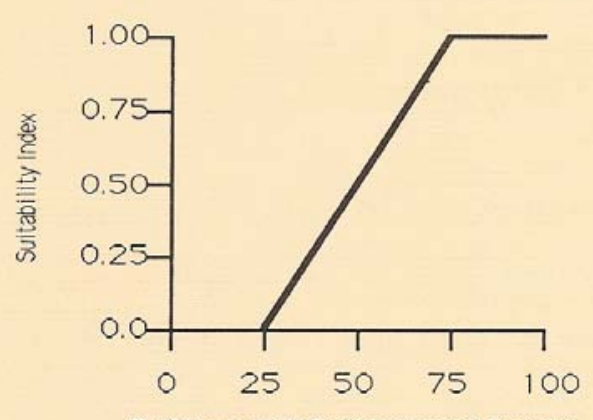

$\%$ of Year with Surface Water Present

\section{$\underline{\text { V1 Field Values }}$}

$\begin{array}{lll}0-25 \% & = & 0.0 \\ 26-50 \% & = & 0.25 \\ 51-75 \% & = & 0.75 \\ >75 \% & = & 1.0\end{array}$

Variable 2: \% Tree canopy cover

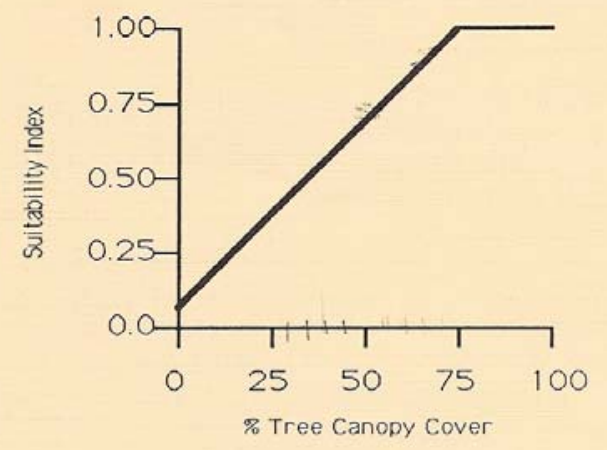

Variable 3: \% Shrub canopy cover

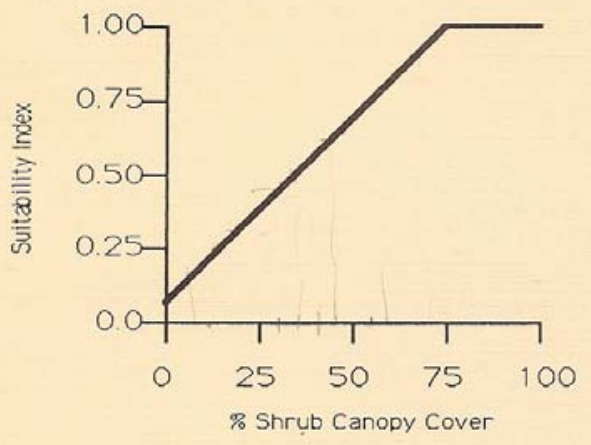

Variable 4: \% Canopy cover of emergent vegetation

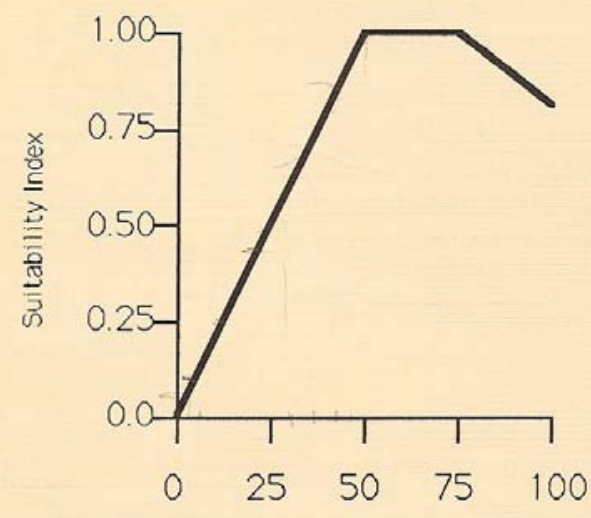

\% Canopy Cover of Emergent vegetation

\section{V4 Field Values}

$\begin{array}{lll}0 \% & = & 0.00 \\ 1-25 \% & = & 0.25 \\ 26-50 \% & = & 0.75 \\ 51-75 \% & = & 1.00 \\ 76-100 \% & = & 0.90\end{array}$


Variable 5: \% Canopy cover of trees and shrubs within $100 \mathrm{~m}$ of wetland edge

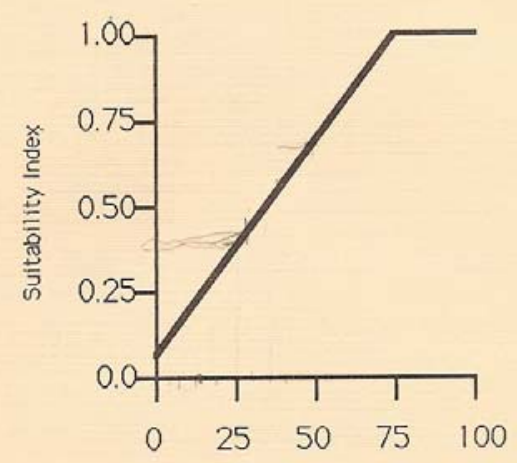

\% Canopy Cover of Trees \& Shrubs $100 \mathrm{~m}$ from Wetland

Variable 6: \% Canopy cover along shoreline

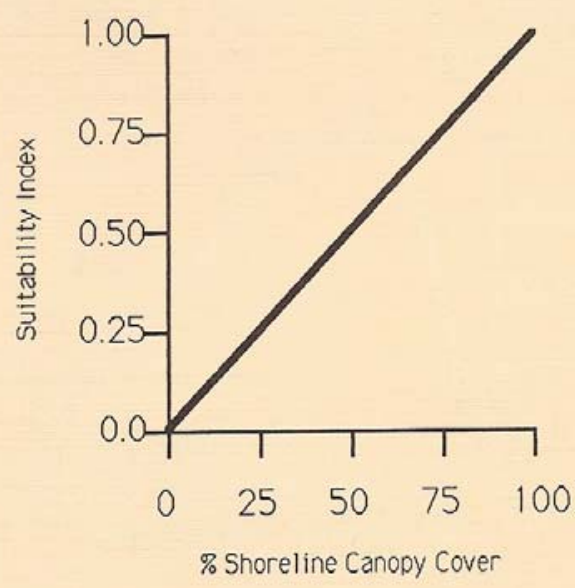

V6 Field Values

$\begin{array}{lll}0 \% & = & 0.00 \\ 1-20 \% & = & 0.10 \\ 21-50 \% & = & 0.35 \\ 51-80 \% & = & 0.65 \\ 81-99 \% & = & 0.90 \\ 100 \% & = & 1.00\end{array}$

In Riparian Forest and Riparian Shrub:

$\begin{aligned} \text { Water SI } & =V 1 \\ \text { Cover SI } & =\frac{M I N\left(1.0\left(V_{2}+V_{3}+V 4\right)+V 5\right.}{2} \\ & \geq \frac{1.0:\left(v_{2}+v_{3}+v_{4}\right)+V_{5}}{}\end{aligned}$

In Emergent Wetlands:

2

$$
\begin{array}{ll}
\text { Water SI } & =\mathrm{V} 1 \\
\text { Cover SI } & =\frac{(4 \times \mathrm{V} 4)+\mathrm{V} 5}{5}
\end{array}
$$

In Riverine and Sand/Gravel/Cobble/Mud Shoreline:

$$
\begin{array}{lll}
\text { Water SI } & =\mathrm{V} 1 \\
\text { Cover SI } & =\quad(\mathrm{V} 5 \times \mathrm{V} 6)^{1 / 2}
\end{array}
$$

Mink HSI = Lowest Value for either Water SI or Cover SI.

Notes: Variables 2, 3, 5 were estimated from $1: 20,000$ color aerial photographs using the continuous variable functions; all other variables estimated at the field sampling sites using the field scales. 


\section{Appendix B - Measurement Techniques}

\section{HABITAT EVALUATION PROCEDURES}

STANDARD MEASUREMENT PROTOCOLS AND TECHNIQUES (Draft)

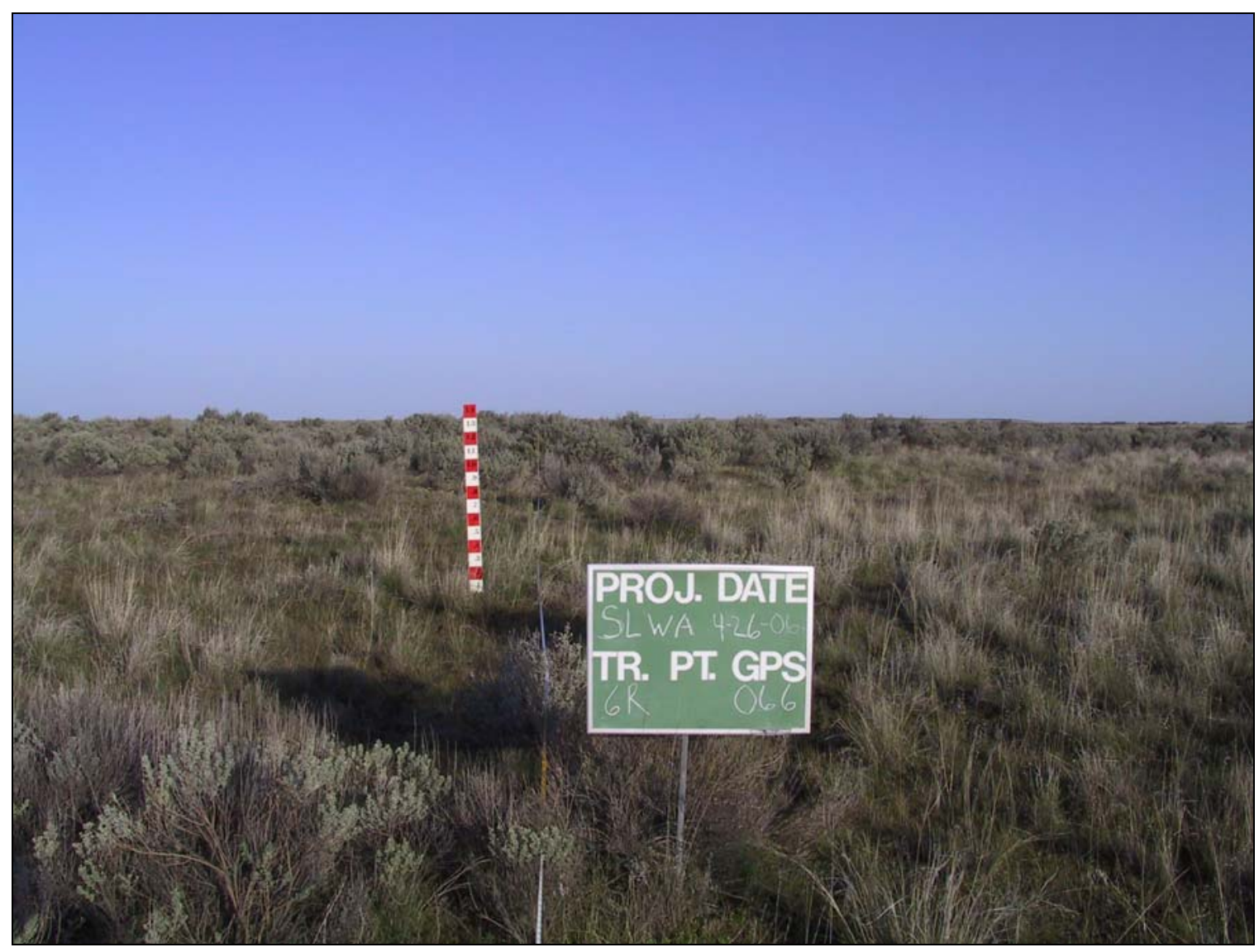

Compiled By

Paul R Ashley - RHT Coordinator

November 2006 
Shillapoo WA 2007 HEP Report

\section{HEP Sampling Design and Measurement Protocols}

\section{Introduction}

This document was developed to fulfill a request by the Upper Columbia United Tribes (UCUT) and Bonneville Power Administration (BPA) to develop a "stand alone" reference for Habitat Evaluation Procedures (HEP) transect protocols used by the Regional HEP Team (RHT). General and specific protocols are described. General protocols include a brief description of pre HEP survey pilot studies; transect establishment guidelines, and photo documentation parameters. In contrast, specific metrics detail actual habitat variable measurement techniques including diagrams where additional explanation is needed.

Specific metrics are identified with an alpha-numeric code. This allows project managers and others to identify specific measurement techniques in report tables without lengthy, redundant explanations. This report is intended to be a "living" document and will be modified as needed. The following standardized protocols and measurement techniques are used by the Regional HEP team to measure habitat variables described in HEP models.

\section{General Protocols}

\section{Pilot Studies}

Pilot studies are conducted in new habitat types and/or familiar habitat types that are comprised of unique structural conditions/key ecological correlates. Pilot study data is used to estimate the sample size needed for a confidence level $\geq 80 \%$ with a $10 \%$ tolerable error level (Avery 1994) and to determine the most appropriate sampling unit ${ }^{6}$ for the habitat variable of interest i.e., a coefficient of variation analysis (BLM 1998). In addition, a power analysis is conducted on pilot study data (and periodically throughout data collection) to ensure that sample sizes are sufficient to identify a minimal detectable change of $20 \%$ in the variable of interest with a Type I error rate $\leq 0.10$ and $\mathrm{P}=0.9$ (BLM 1998, Block et al. 2001). All field data is recorded on data loggers or data sheets and downloaded/transferred to data summary spreadsheets.

Transects

Transect cover sheets are used to document specific transect information including transect identification, cover type, HEP Team members, global positioning system (GPS) coordinates, and other pertinent information.

Transects are established at least 300 feet (100 meters), where possible, from ecotones, roads, and other anthropogenic influences. Transect starting points and azimuths (direction) are randomly selected for each cover type. Start points are selected based on superimposing a UTM grid over cover type maps and identifying specific X/Y coordinates with the aid of a random numbers table, or computer generated random number generator/point locater program.

\footnotetext{
${ }^{6}$ Includes micro-plot grid size and shape etc.
} 
Transect start, turn, and end points are marked with 14 -inch (36 centimeter) 0.25 inch ( 0.6 centimeter) diameter rebar stakes ${ }^{7}$ painted fluorescent orange or red. GPS positions (UTM coordinates-NAD 27) are recorded at start, turn, and end points. If cover types change or transect length is greater than 300 feet, another transect azimuth is randomly selected, or the original azimuth is varied by 45 degrees (direction [left or right] is determined by the flip of a coin where more than one choice is possible). Compass azimuths (headings) are magnetic bearings i.e., not corrected for local declination. Transects are divided into 100 foot (30 meter) sample units for statistical purposes.

\section{Photo Points}

Photo points are established at the start point of each transect. Pictures are recorded from a height of three feet at the beginning of each transect while facing in the direction of the transect azimuth. A transect reference board (includes transect number, project name, date, GPS reference number) is placed at the 15 foot interval while a cover board is placed at the 30 foot mark on each transect. Occasionally, panoramic photographs are also needed e.g., dense vegetation, linear/narrow cover types. Habitat conditions are photographed with a Canon G1 ${ }^{\circledR} 3.3$ mega pixal digital camera (with and without magnification).

\section{Specific Metrics}

Metrics generally follow those described by Hays et al. (1981) and/or Avery (1994) unless otherwise noted. Some metrics have been modified due to extreme field conditions and/or to better meet Regional HEP Team needs.

\section{Herbaceous Measurements}

\section{Percent Cover}

1. Herbaceous percent cover measurements are recorded at 20 or 25-foot intervals on the right side of the transect tape (the right side is determined by standing at 0 feet and facing the line of travel/transect azimuth). RHT members walk on the left side of the transect line to reduce sample disturbance. A square $0.1 \mathrm{~m}^{2}$ micro-plot grid is used in grasslands to estimate percent cover of herbaceous vegetation while a rectangular $0.5 \mathrm{~m}^{2}$ grid is generally used in shrublands (the $0.5 \mathrm{~m}^{2}$ grid may also be used in grasslands if desired). The near right hand corner of the grid is placed at the sampling interval (rectangle grids are placed with the long axis perpendicular to the tape, and the lower right corner on the sampling interval). An example of micro-plot grid placement is shown in Figure 1 . Approximately $20 \%$ of the micro plot is covered by vegetation in the example. Grid samples are considered independent samples for statistical purposes.

1A: $0.1 \mathrm{~m}^{2}$ micro-plot grid/20' interval

\footnotetext{
${ }^{7}$ Marking transect points with rebar stakes is at the discretion of the project proponent. Therefore, not all transects are marked in this manner.
} 
1B: $0.1 \mathrm{~m}^{2}$ micro-plot grid $/ 25$, interval

$1 \mathrm{C}: 0.5 \mathrm{~m}^{2}$ micro-plot grid/20' interval

1D: $0.5 \mathrm{~m}^{2}$ micro-plot grid $/ 25$ ' interval

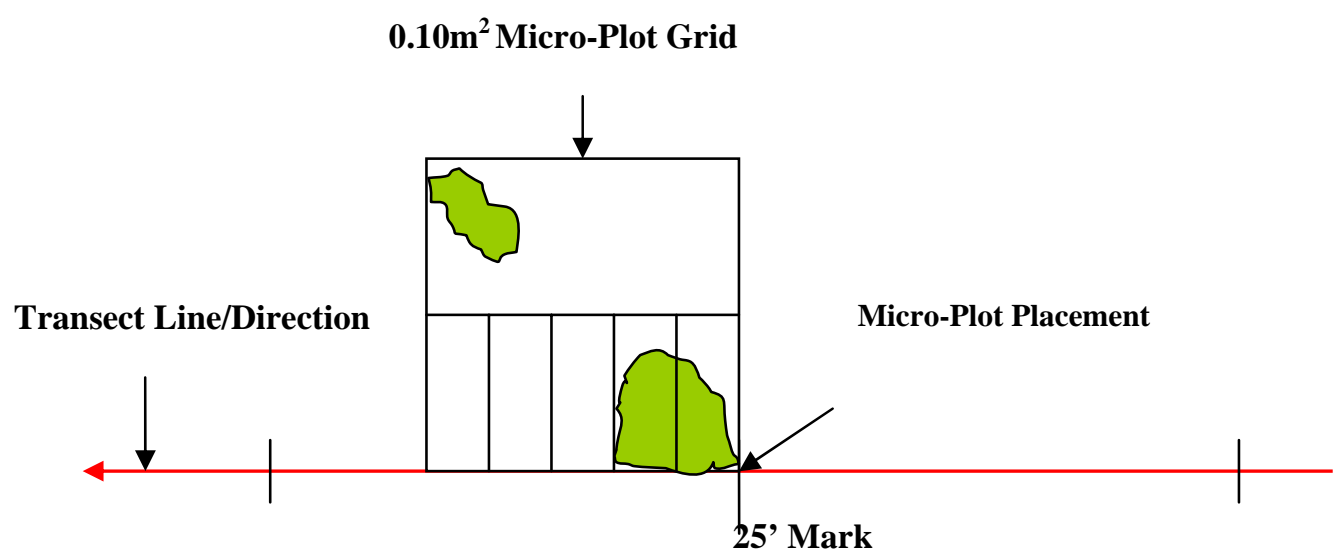

Figure 1. Micro-plot grid placement and percent cover example.

\section{Height}

2. Herbaceous height is measured with a measuring rod placed within the grid frame (scale $=10$ ths $/ \mathrm{ft}$.). Three evenly spaced measurements are recorded and averaged for each sample. Only leaf material is measured (leaves provide the greatest amount of cover). "Leaf material" may include residual cover and/or new growth predicated on HEP model variable requirements. Grass inflorescence is not included in height measurements.

2A. Four measurements, one from each corner of the micro plot grid, are recorded and averaged for each sample. Only leaf material is measured (leaves provide the greatest amount of cover). Grass inflorescence is not included in height measurements.

2B. A measuring rod is held vertical at the interval point: the highest vegetation to cross the measuring rod at that point is measured to the nearest tenth of a foot.

2B-1: 10' interval

2B-2: 20' interval

2B-3: $25^{\prime}$ interval

\section{Visual Obstruction Readings (VOR)}


3. A Robel pole (Robel 1975) is used to document vertical and/or horizontal cover for herbaceous vegetation i.e., visual obstruction readings (VOR). Measurements are recorded at 20, 25, or 50-foot intervals. Intervals are determined by the length of each transect, i.e., a minimum of 12 measurements are required for each transect, or cover type heterogeneity (structurally diverse cover types generally require larger sample sizes).

The Robel pole (Robel 1975) is placed on the transect line at the appropriate interval. Four observations are taken from a distance of four meters from the Robel pole and averaged to obtain a single visual obstruction reading or VOR. Observers sight over a one meter pole and record how much of the Robel pole is totally obscured from the ground up (Figure 2). Measurements are reported in 0.25 decimeter increments.

Two measurements are taken on the transect line on opposite sides of the Robel pole; two identical measurements are taken from the same point perpendicular to the transect line for a total of four "readings" (Figure 3). Sample size is determined to be adequate when the "running mean" varies $\leq 10 \%$ of the mean. VOR samples are considered independent for statistical purposes.

3A: 20 ' interval

3B: 25 interval

$3 \mathrm{C}: 50$ ' interval

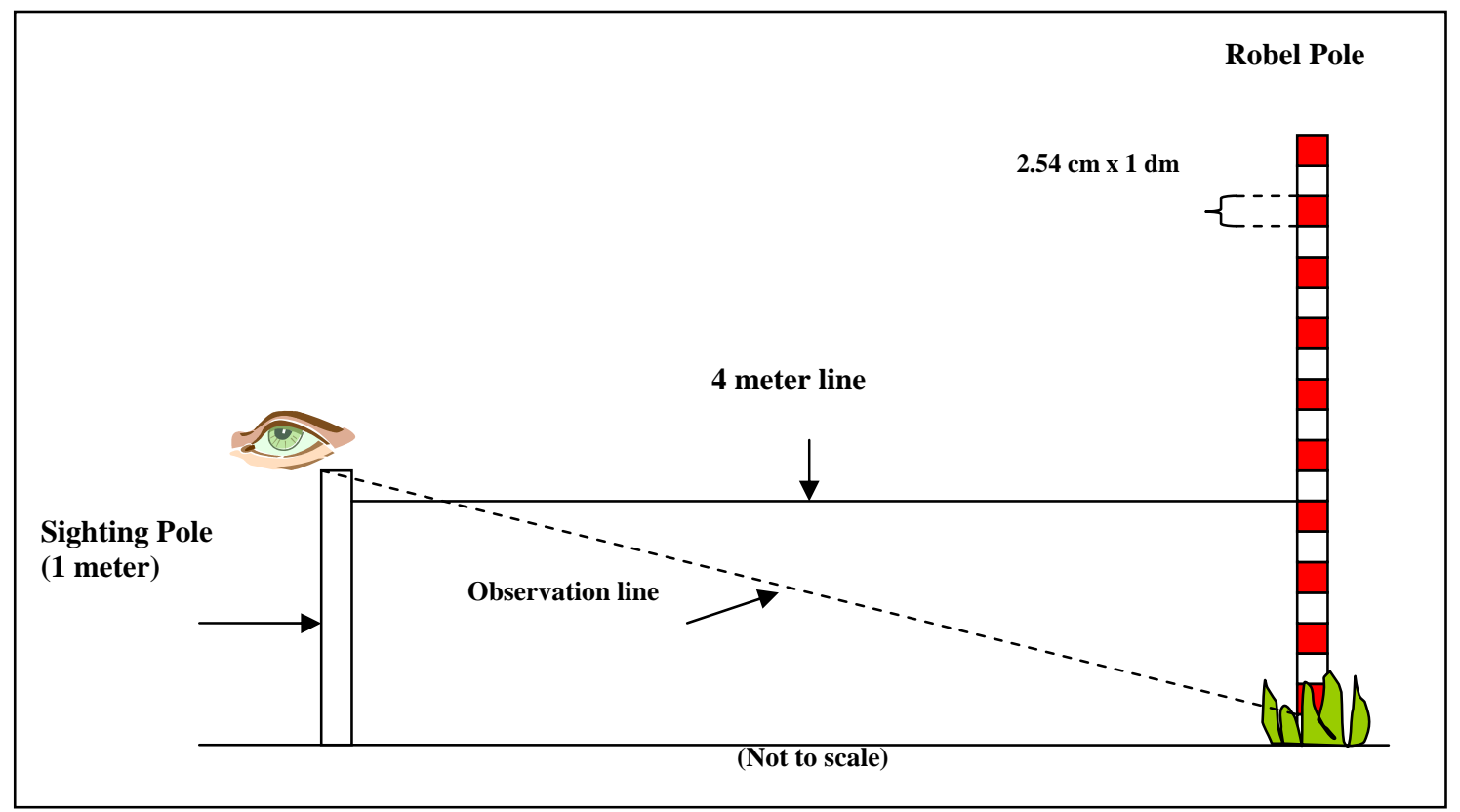

Figure 2. Visual obstruction reading diagram. 


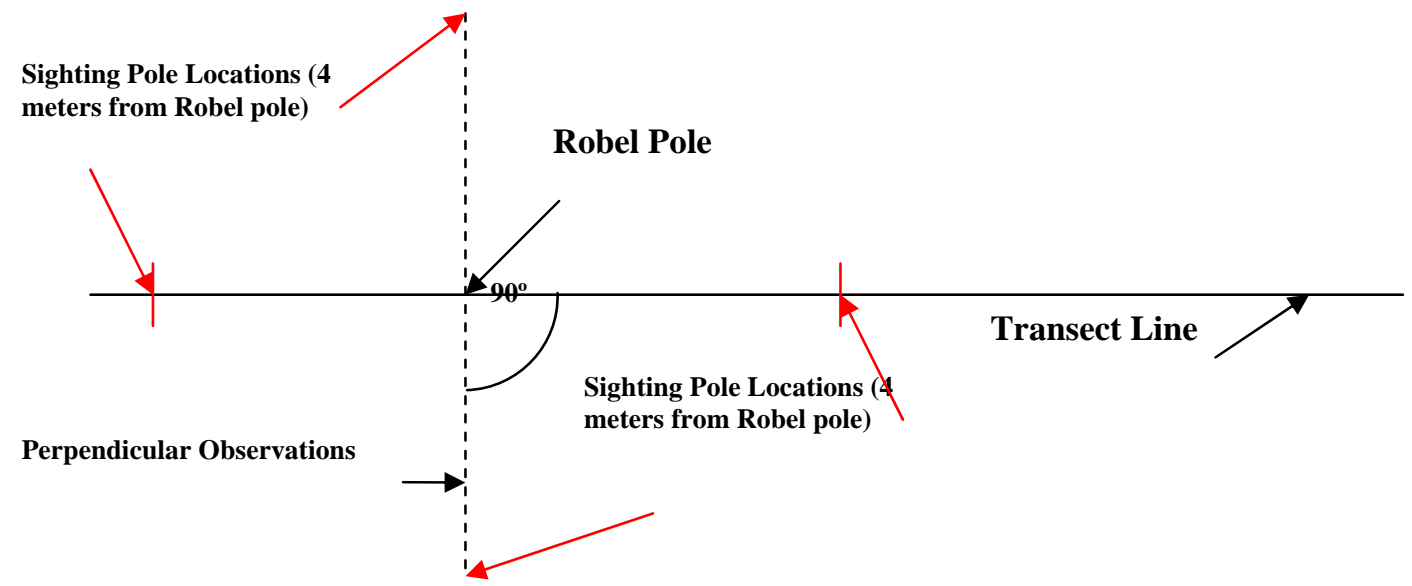

(“Birds eye” View)

Figure 3. Robel pole "readings" layout diagram.

\section{Shrub Measurements}

\section{Percent Cover}

4. Line intercept or point intercept (USFWS 1981) is used to determine shrub cover. Line intercept is generally used when shrub cover is estimated at $<5 \%$ (the most accurate results are obtained using the line intercept method). In contrast, the point intercept method is used if shrub cover is estimated at $>5 \%$.

4A: Line intercept is used to measure the amount of cover that intercepts the transect line as illustrated by the red lines shown in Figure 4. Measurements are in $10^{\text {ths }}$ of feet. Gaps in vegetation less than four tenths of a foot (5 inches) are ignored. The amount covered by shrubs is added to determine shrub intercept for each transect. For example, if 7.5 feet of a 100 -foot long transect is covered by shrubs, percent cover is $7.5 \%$.

Shrub cover is recorded by species. Where shrubs overlap, shrub intercept is recorded for the tallest shrub and noted for the lower shrub(s). 


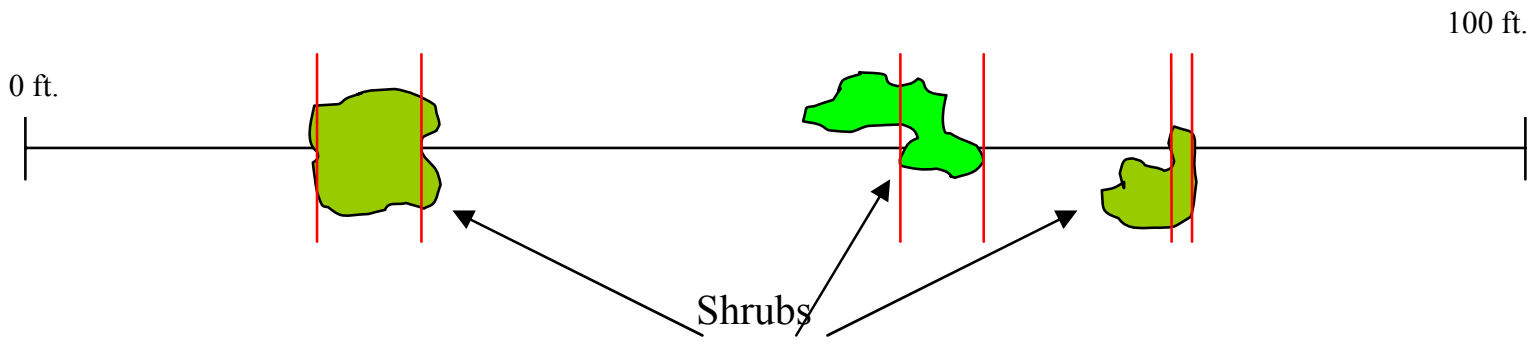

Figure 4. Line intercept method example.

4B: Point intercept is used when shrub canopy cover is estimated at $\geq 5 \%$.

Shrub cover is determined by recording the number of "hits" at specific intervals along a transect line. To be counted as a "hit", a portion of the shrub must cross the transect tape's interval number line e.g., 2', 4', 6'... nth. If a portion of the shrub does not break the vertical plane at the interval number line, it is reported as a miss (Figure 5). Either a "hit" or "miss" is recorded on data loggers and/or paper data sheets for each designated interval.

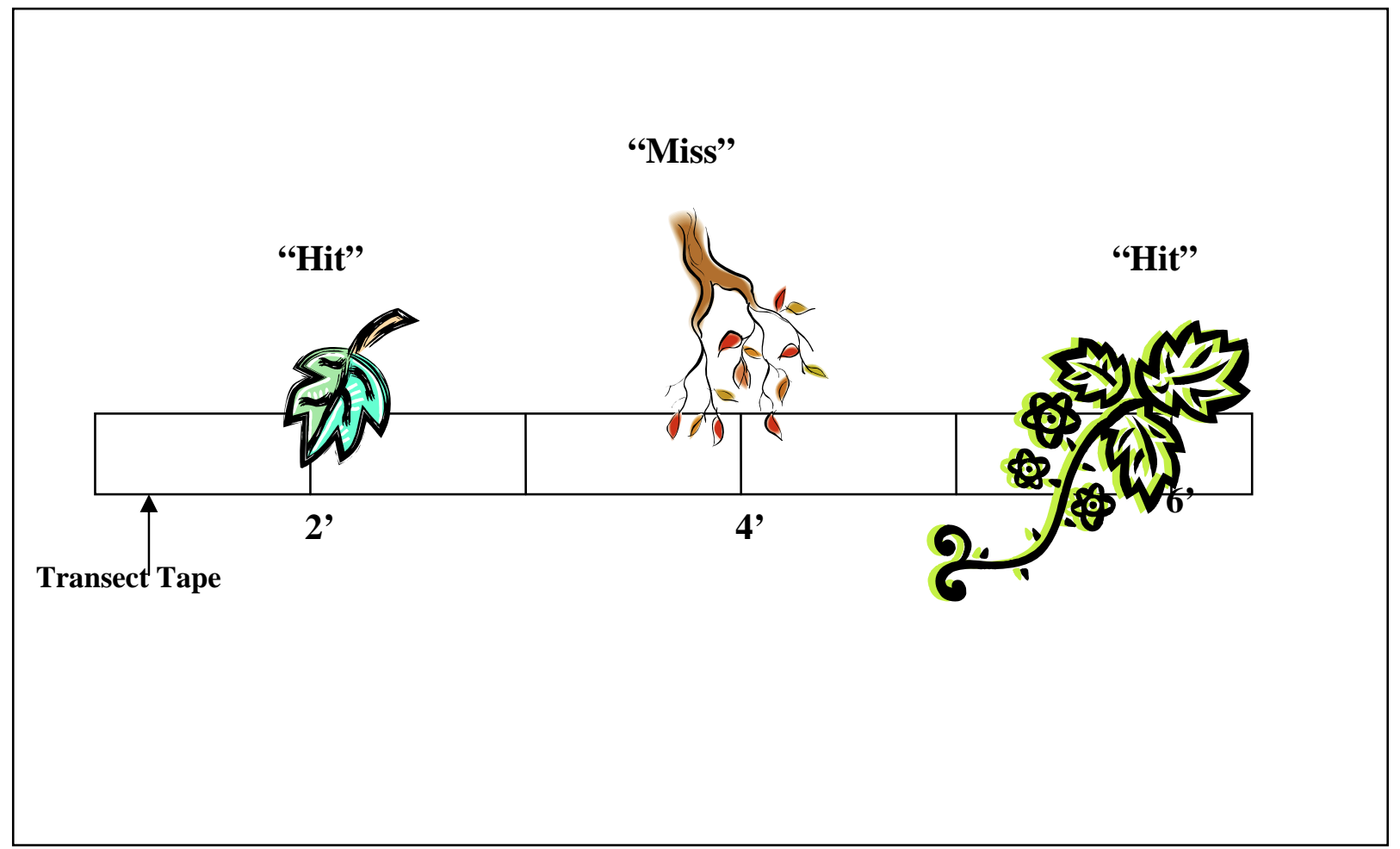

Figure 5. Point intercept method example showing "hits" and "misses" at two foot intervals. 
From $5 \%$ to $20 \%$ cover, point data is collected at two-foot intervals ( 50 possible "hits" per $100 \mathrm{ft}$. sample unit). If shrub cover is estimated at $>20 \%$, shrub point data is collected at five foot intervals (20 possible "hits" per 100 $\mathrm{ft}$. sample unit). On rare occasions, ten-foot intervals may be used when shrub cover exceeds $50 \%$ (10 possible "hits" per $100 \mathrm{ft}$. sample unit). The ten-foot interval is generally applied to shrub monocultures, or areas with few shrub species that exhibit relatively equal shrub distribution/density.

Shrub "hits" are recorded by species. Where shrubs overlap, shrub intercept is recorded for the tallest shrub and noted for the lower shrub(s).

4B-1: 2' interval

4B-2: 5 ' interval

4B-3: 10' interval

4C: Modified point method is used when shrub cover is impenetrable or otherwise inaccessible. A baseline transect is established along the shrub edge. A six-foot measuring rod is then inserted into the shrub cover at right angles to the baseline tape at appropriate intervals. Recorders estimate shrub "hits", species information, and height data where the end of the six-foot measuring rod intercepts the shrub cover (Figure 6). As with point intercept, intervals may very. Shrubs are identified by species.

4C-1: 2' interval

4C-2: 5 ' interval

4C-3: 10' interval

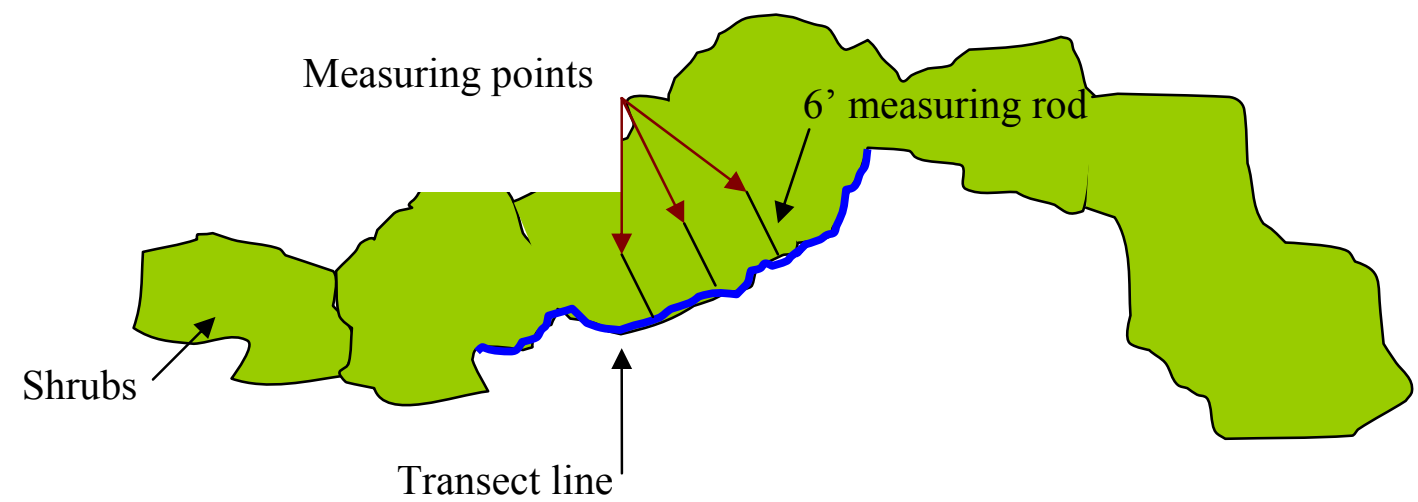

Figure 6. Modified point intercept layout example. 
4D: Complex shrub intercept is used to determine percent shrub cover in multi strata shrub communities. This method is generally associated with point intercept methods whereas overlapping shrubs are identified for each stratum. Percent cover is determined for each of four possible strata as well as total percent shrub cover and overlapping percent cover.

The complex shrub intercept method is identified by adding the suffix "4D" after the appropriate line or point intercept method. For example, "4B-1-4D designates that complex shrub point intercept measurements were taken at two foot intervals. Similarly, 4C-2-4D designates that modified point intercept at five foot intervals was used to determine percent shrub cover for strata in a complex shrub community.

\section{Shrub Height}

5. Shrubs are defined as woody vegetation including trees $<16$ feet in height unless otherwise defined in HEP models. The Regional HEP Team assumes that trees $<16$ feet tall function ecologically more like shrubs than trees.

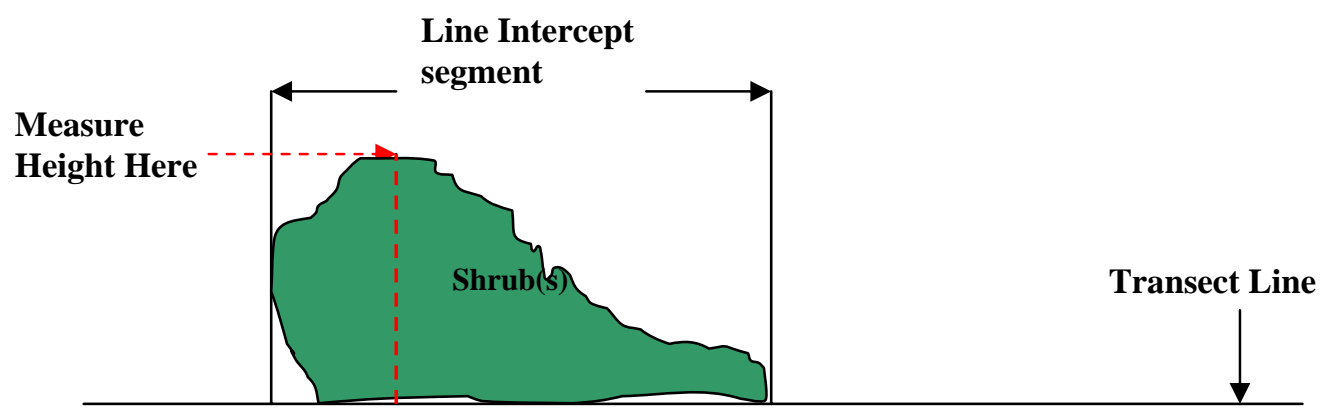

Horizontal View

Figure 7. Line intercept shrub height measurement example.

Shrub height is measured in $10^{\text {ths }}$ of feet at the highest point for each uninterrupted line intercept segment as depicted in Figure 7, or the highest point that crosses each point intercept interval mark on the transect tape (Figure 8).

In structurally complex (overlapping) shrub communities, height is measured for each stratum (maximum of four) as illustrated in Figure 9. It is assumed that shrub height measurements correspond to the method used to determine percent shrub cover. For example, if percent shrub cover is determined using the line intercept 
method (Figure 4), then it is assumed that shrub height will be obtained as illustrated in Figure 7.

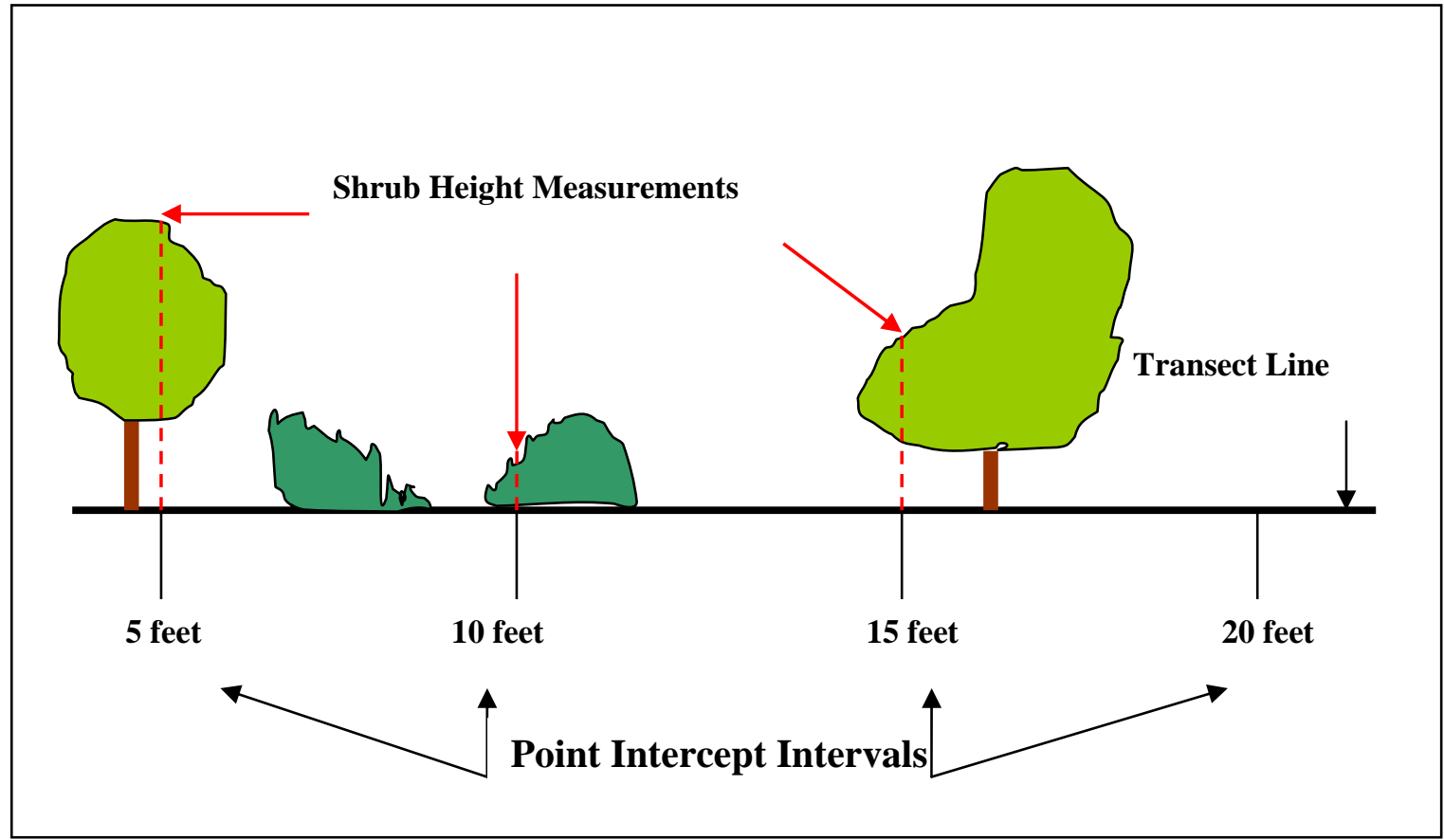

Figure 8. Point intercept shrub height example.

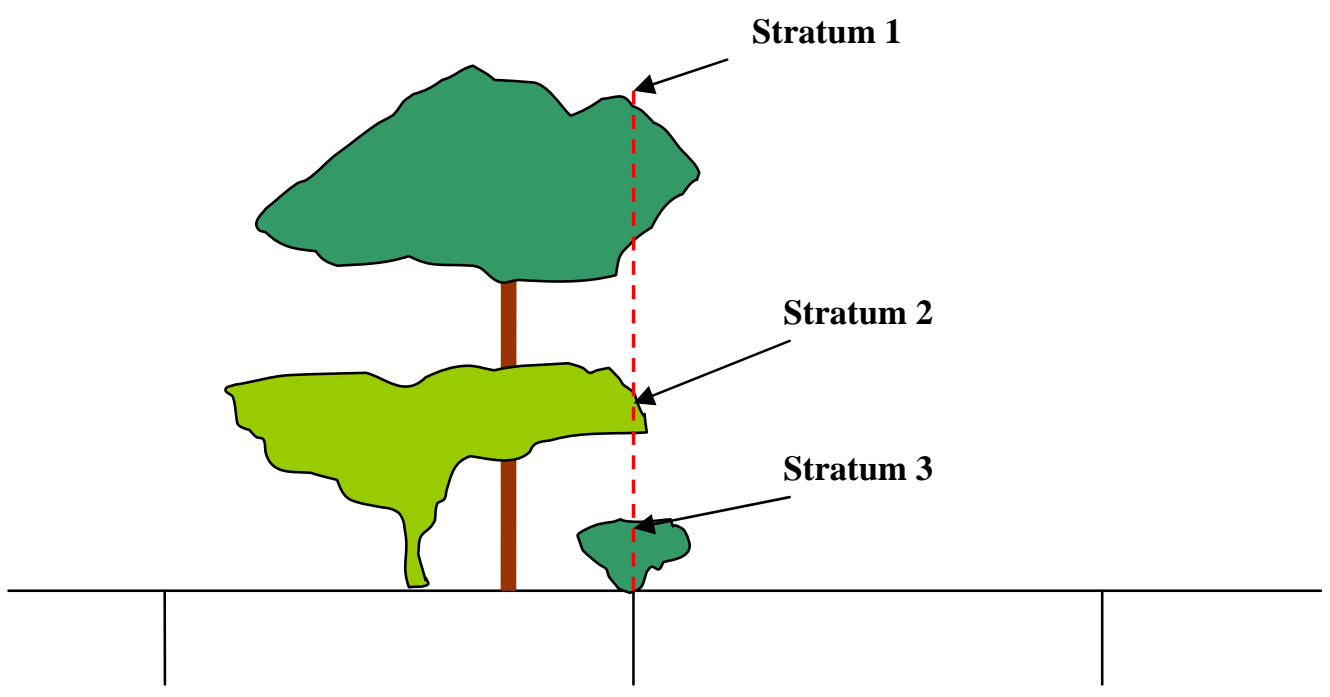

Figure 9. Complex shrub community shrub height measurement example. 


\section{Tree Measurements}

\section{Percent Canopy Cover}

6. Tree canopy cover measurements are recorded at five or ten foot intervals with a densitometer (point intercept). Measurement intervals are determined by visually estimating tree canopy closure prior to initiating the survey. If estimated canopy closure is $<20 \%$ and estimated transect length $\leq 900$ feet, measurements are recorded at five-foot intervals; if estimated canopy closure is $>20 \%$ and estimated transect length is $\geq 600$ feet, ten-foot intervals are used. The size of the sample area strongly influences transect length. In small areas, data from several short (300 foot) transects may be "pooled" in order to determine percent tree canopy cover. As with shrubs, sampled trees are identified by species and the sampling unit is a 100 foot segment of the transect.

6A: 5 ' interval

6B: 10' interval

\section{Height}

7. Tree height is determined generally using a clinometer. In open areas, an electronic height measurement instrument may be used. Measurements are taken at the beginning and end of each transect and at 100 foot intervals. Additional samples may be taken if needed. HEP model variable requirements determine the extent of tree height measurements e.g., multi-canopy, overstory, etc.

Basal Area

8. Tree basal area data is collected at 100-foot intervals using a "factor 10" prism. Each 100-foot interval basal area observation (all tree "hits" at each 100-foot point) is considered an independent sample.

\section{Snag DBH}

9. Snag data is collected on belt transects. RHT members collect snag data in conjunction with tree canopy closure measurements using the same baseline transect. The diameter breast height (DBH) of all snags present within tenth-acre belt transects paralleling the baseline transect is measured. Either the actual DBH is recorded, or snag data is reported by class e.g., 5 snags $<4$ " DBH, 2 snags $>20$ " $\mathrm{DBH}$ etc.

Belt transects are 44 feet wide by 100 feet long i.e., 22 feet on each side of the baseline transect. Belt transect layout is depicted in Figure 10. As with shrubs and trees, the sampling unit is each 100-foot segment. 


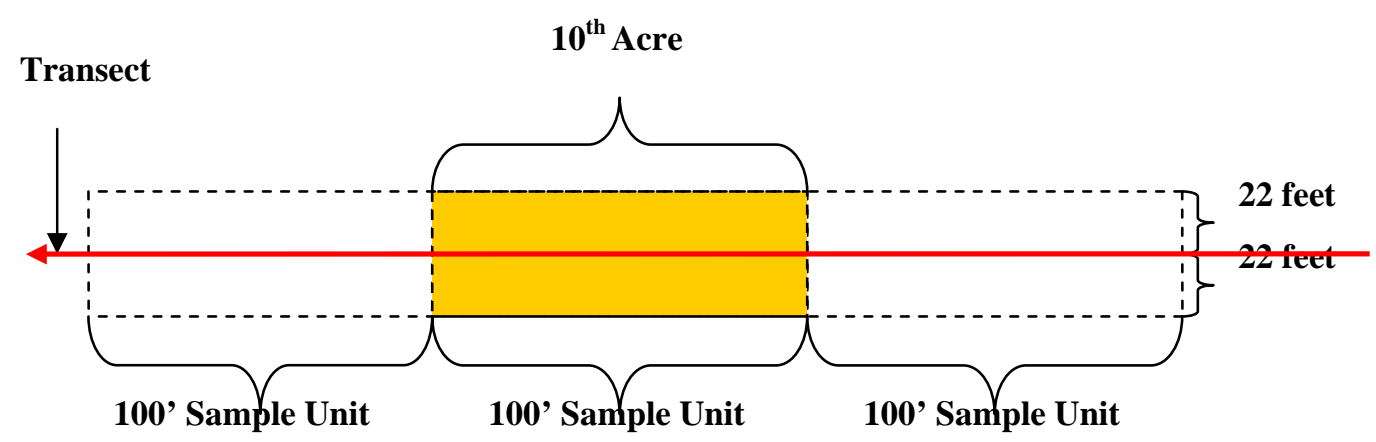

Figure 10. Belt transect layout diagram.

\section{Sample Size Determination}

The process for determining sample size (transect length) varies based on the variable measured. Shrub and tree cover and grid sample sizes are estimated as follows:

The amount of cover within each 100 foot sample unit is divided by sample unit length to obtain percent shrub/tree cover per sample unit (e.g. 10 feet of cover/100 feet $=10 \%$ shrub cover). The standard deviation for each transect is calculated for percent cover data from transect sample units. Sample size (transect length) is then determined through use of the following equation (Avery 1994):

$$
\mathrm{n}=\frac{\mathrm{t}^{2} \mathrm{~s}^{2}}{\mathrm{E}^{2}}
$$

Where: $\mathrm{t}=\mathrm{t}$ value at the 95 percent $(0.05)$ confidence interval for the appropriate degrees of freedom (df); $\mathrm{s}=$ standard deviation; and $\mathrm{E}=$ desired level of precision, or bounds ( \pm 10 percent). Confidence intervals may vary from 80 percent $(0.20)$ to 95 percent $(0.05)$ depending on habitat variable heterogeneity and project management needs. The same method is used to determine sample size for micro plot samples based on total percent cover for herbaceous species. 


\section{References}

Avery, T.E., H. E. Burkhart. 1994. Forest measurements. $4^{\text {th }}$ edition. John Wiley and Sons. New York, NY.

BLM. 1998. Measuring and monitoring plant populations. BLM Technical Reference 1730-1. BLM National Business Center. Denver, CO. 477 p.

Block, W.M., W.L. Kendall, M.L. Morrison, and M. Dale Strickland. 2001. Wildlife study design. Springer Press. New York, NY. 210 p.

Hays, R. L., C. Summers, and W. Seitz. 1981. Estimating habitat variables. Western Energy and land Use Team. Fort Collins, CO: U.S. Fish and Wildlife Service.

Robel, R.J., J. N. Dayton, A.D. Hulbert. 1975. Relationship between visual obstruction measurements and weight of grassland vegetation. Journal of Range Management. 23: 295. 
Shillapoo WA 2007 HEP Report

\section{Appendix C - Transect Photographs}

\section{Transect 1}

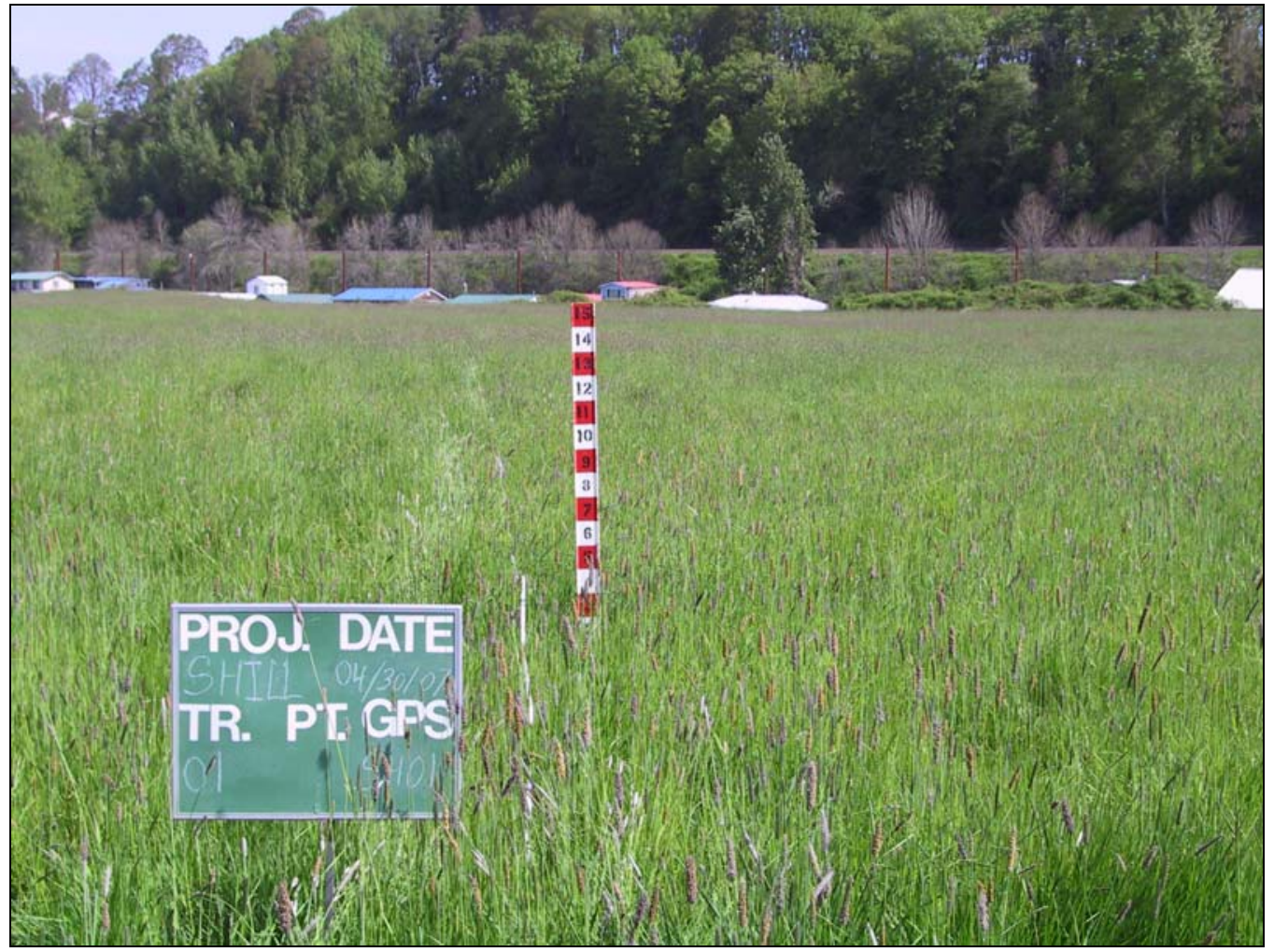


Shillapoo WA 2007 HEP Report

Transect 2

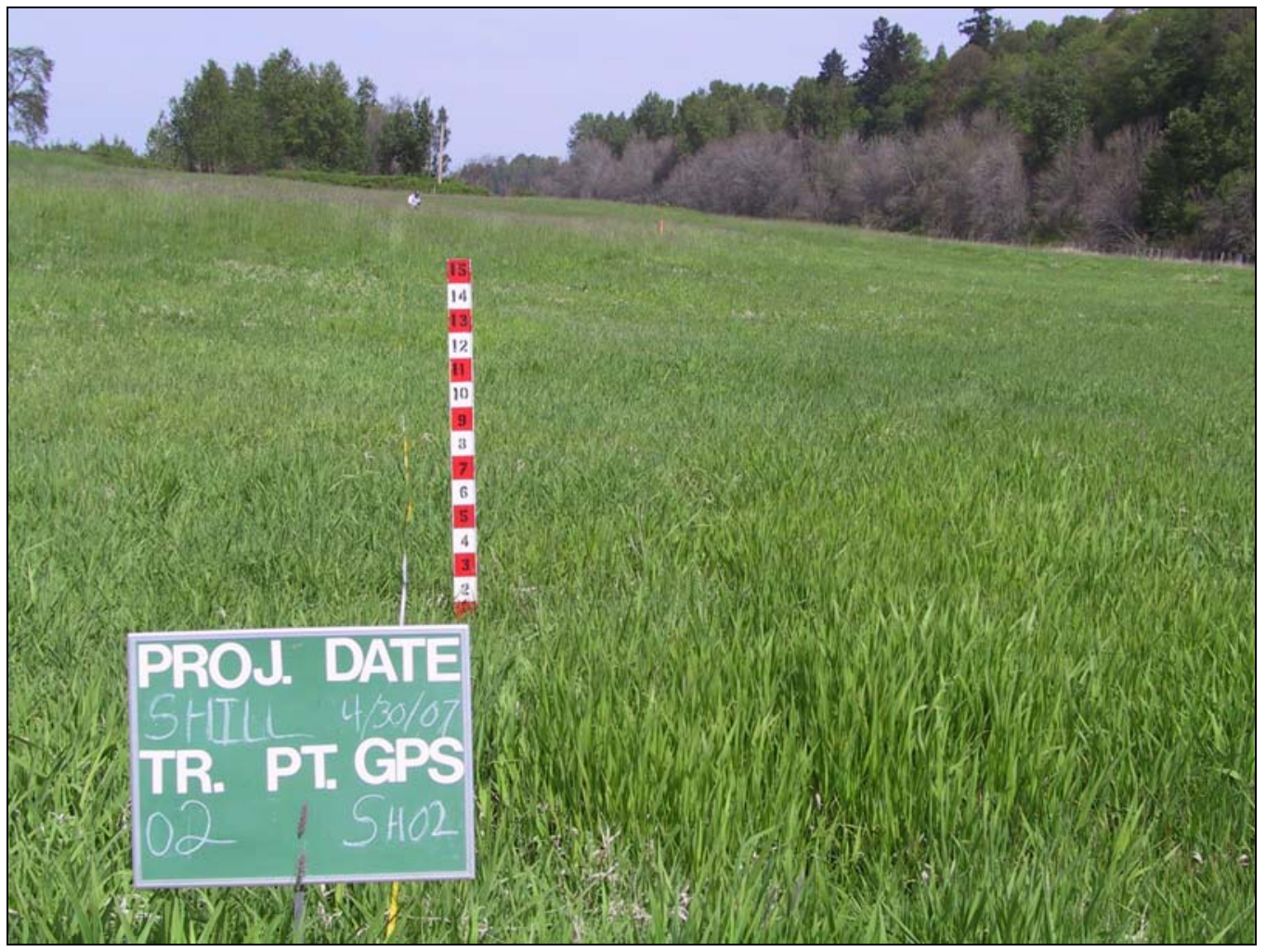

Transect 3

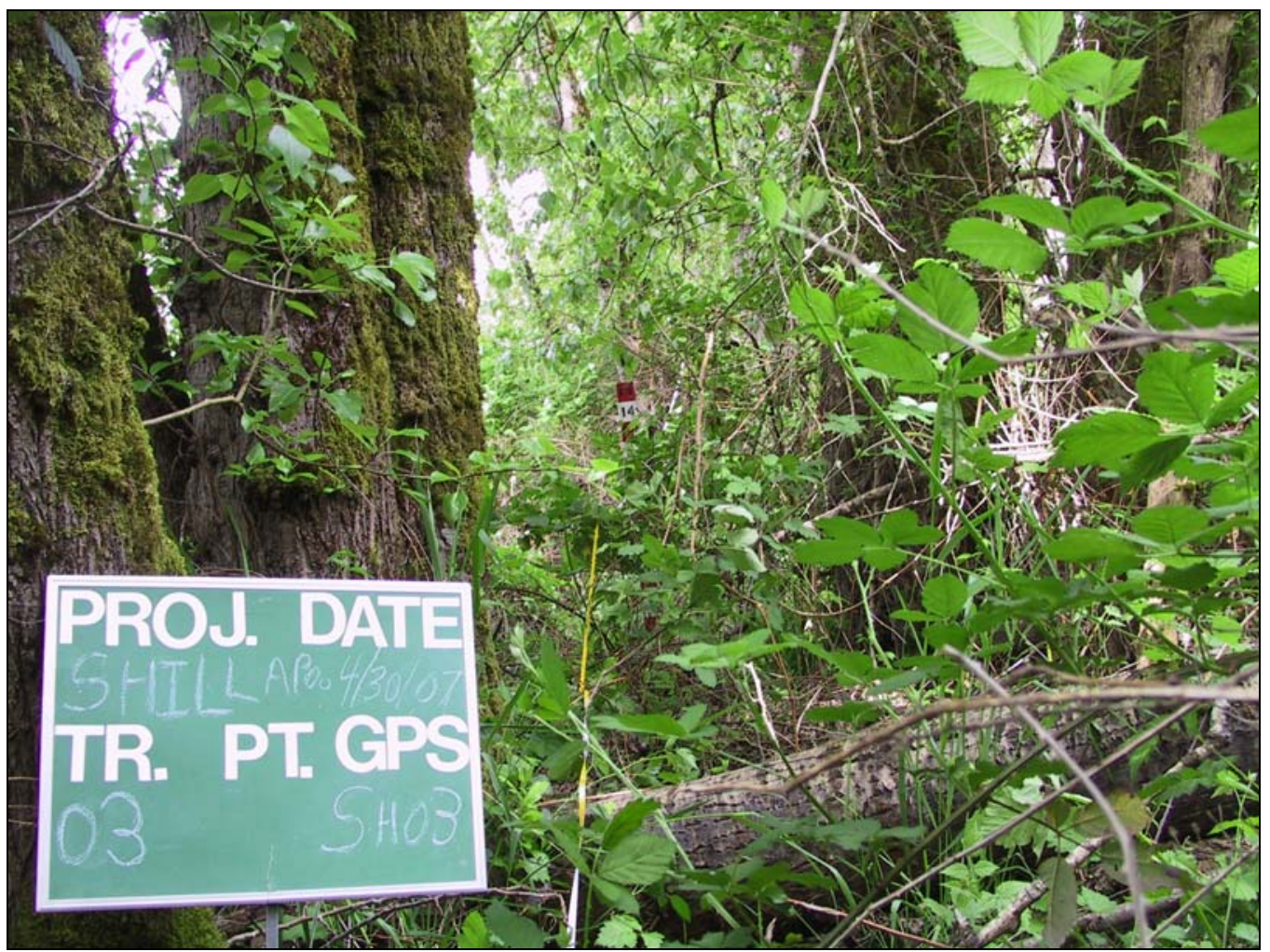


Shillapoo WA 2007 HEP Report

\section{Transect 4}

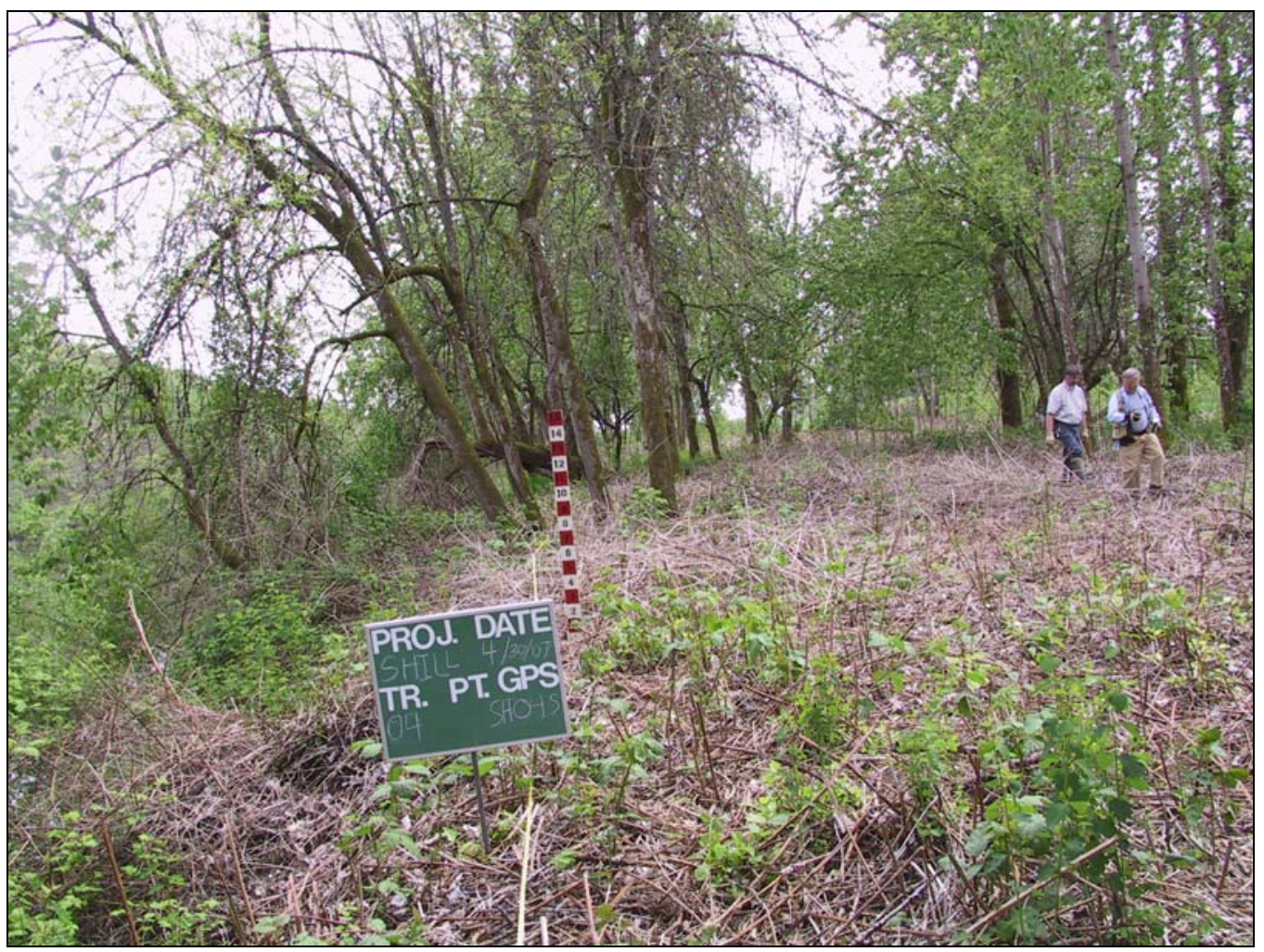

Transect 5

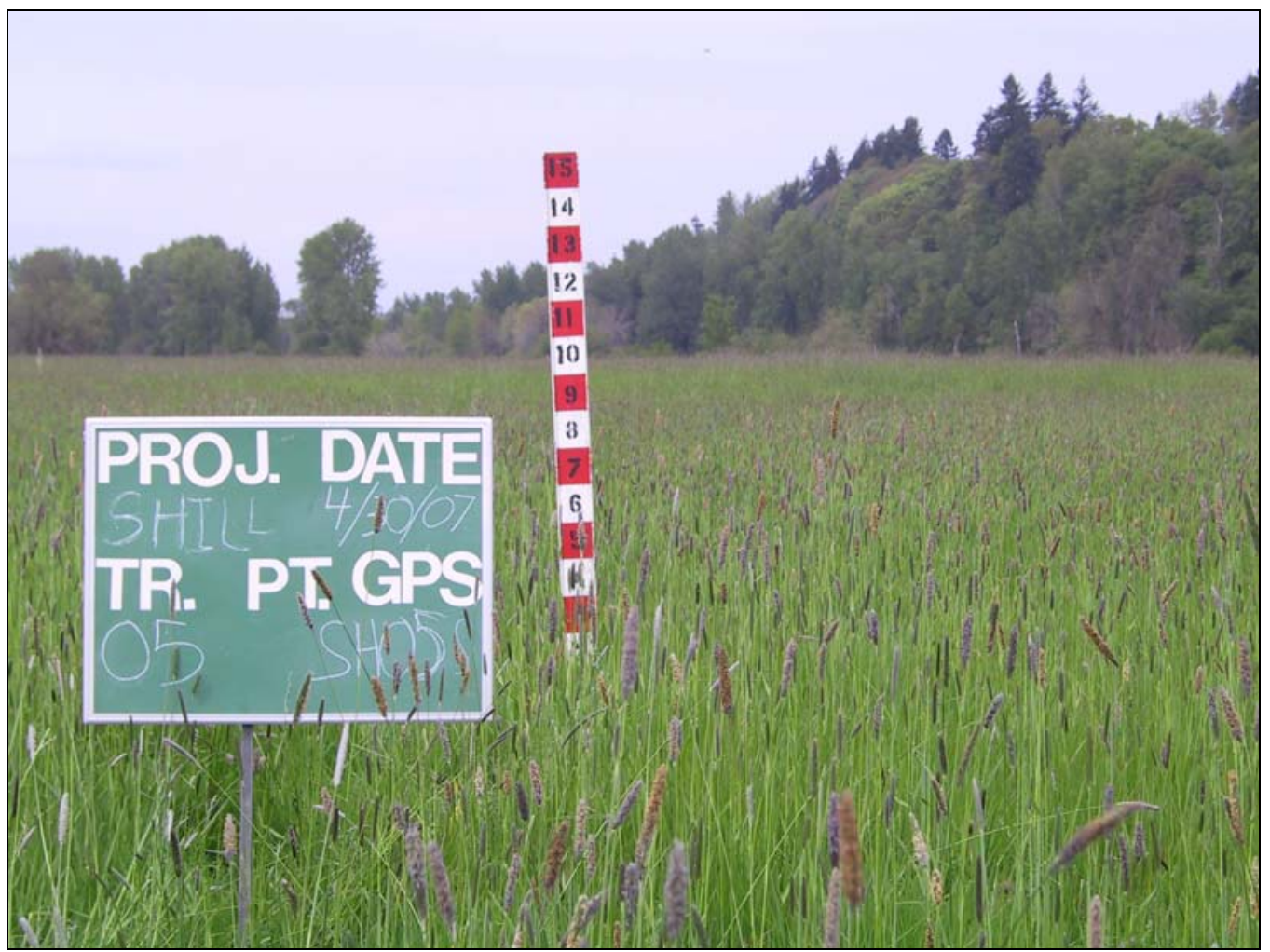


Shillapoo WA 2007 HEP Report

\section{Transect 6}

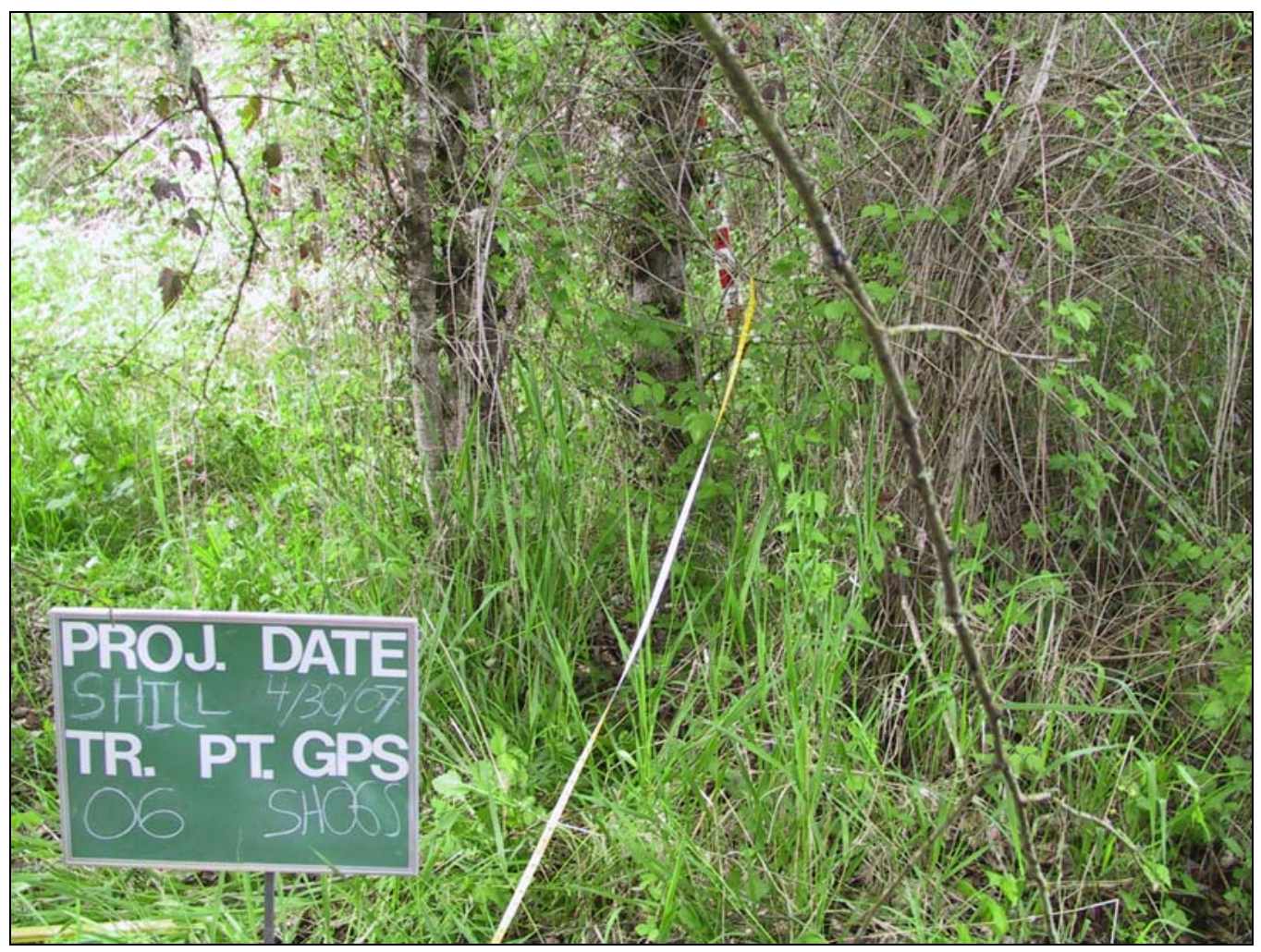

Transect 7

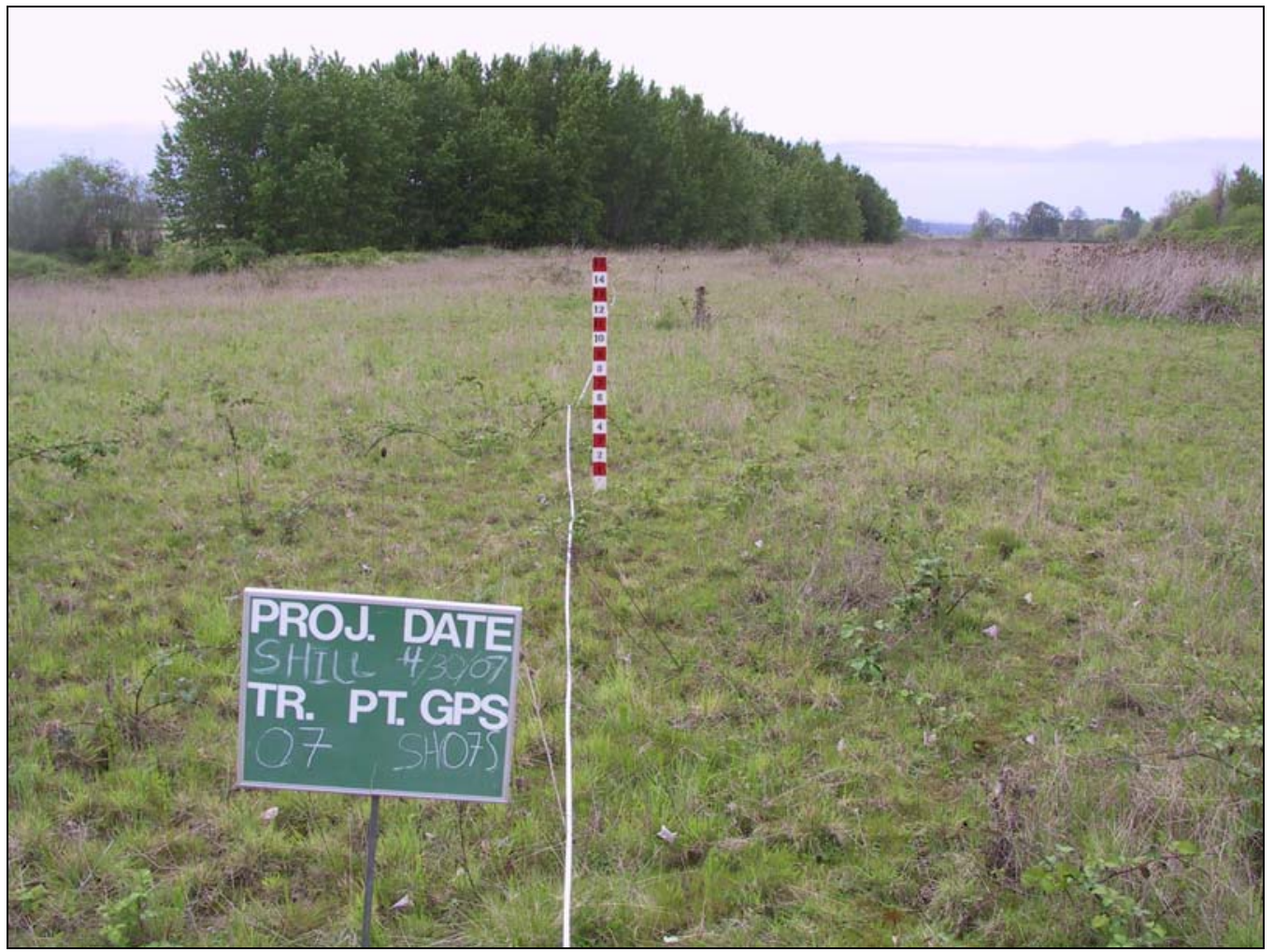


Shillapoo WA 2007 HEP Report

\section{Transect 8}

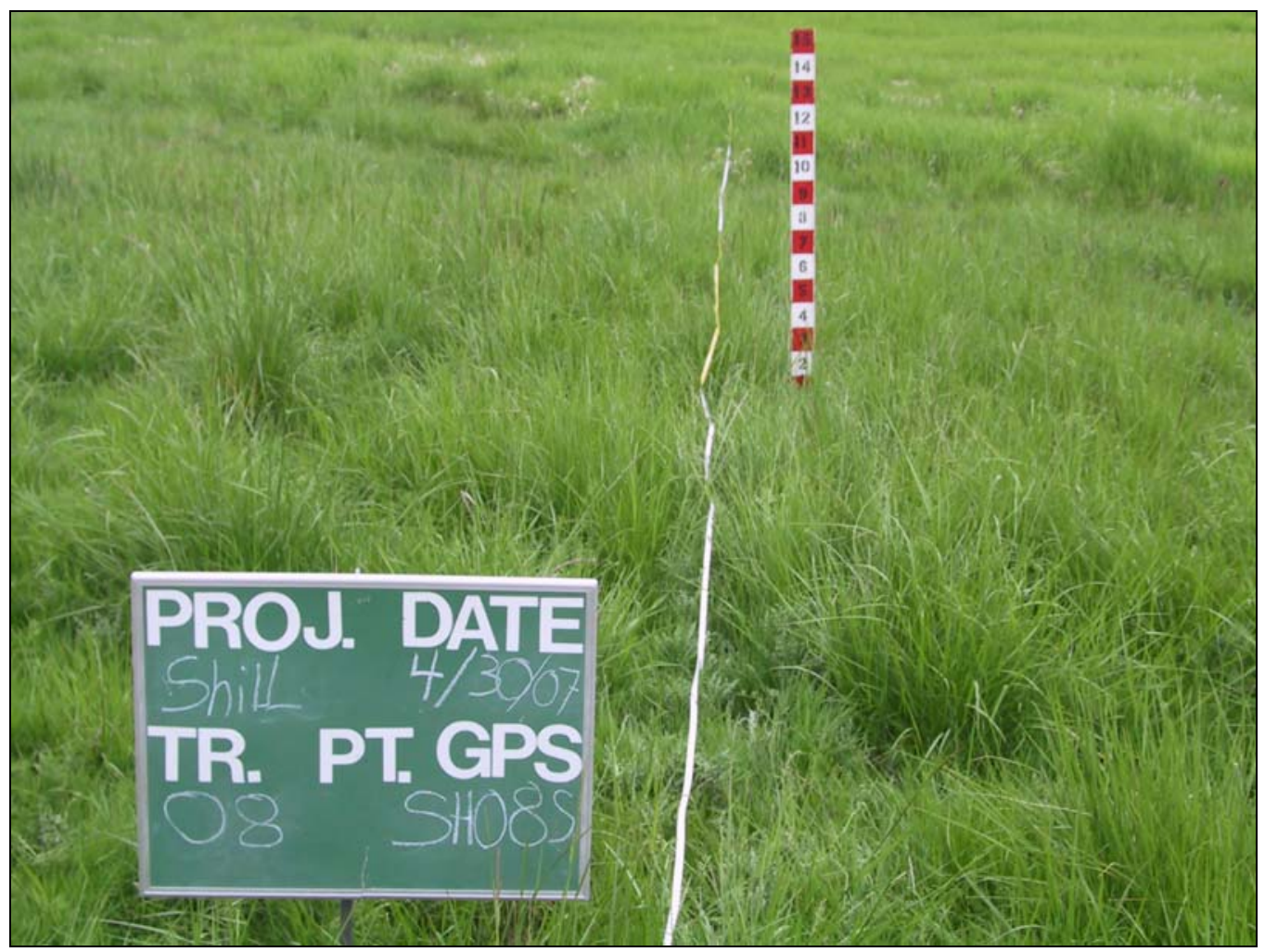

Transect 9

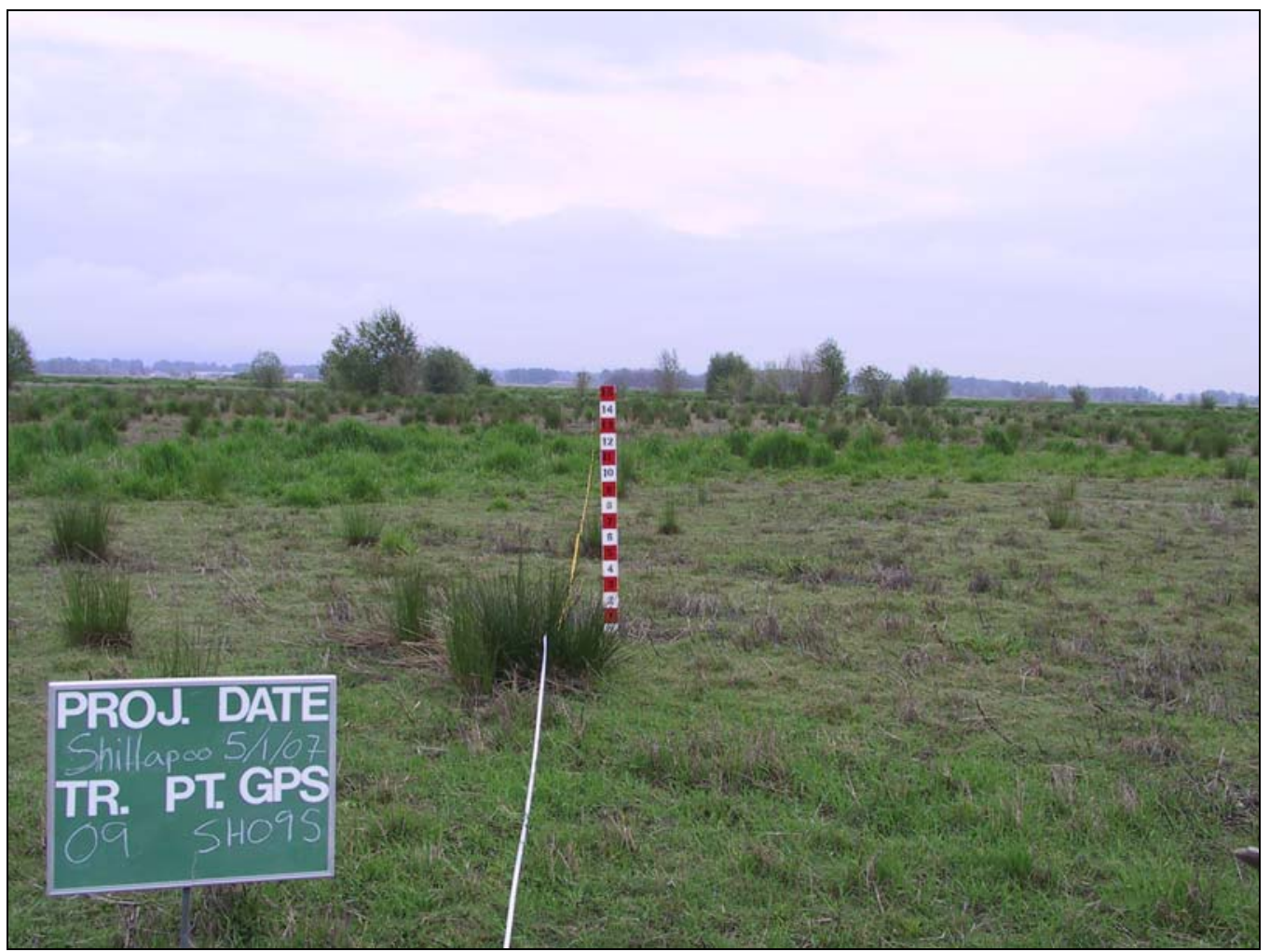


Shillapoo WA 2007 HEP Report

\section{Transect 10}

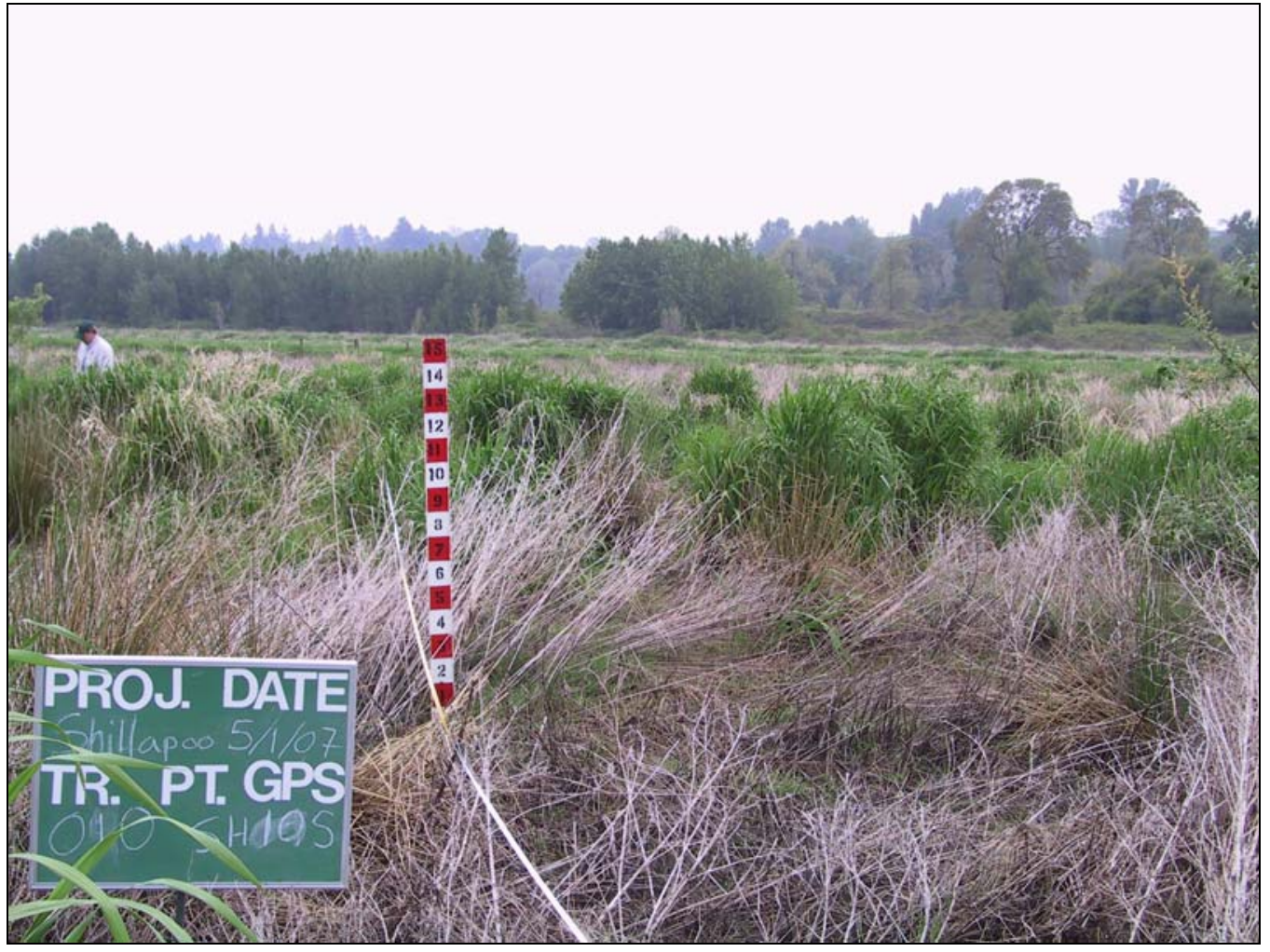

\section{Transect 11}

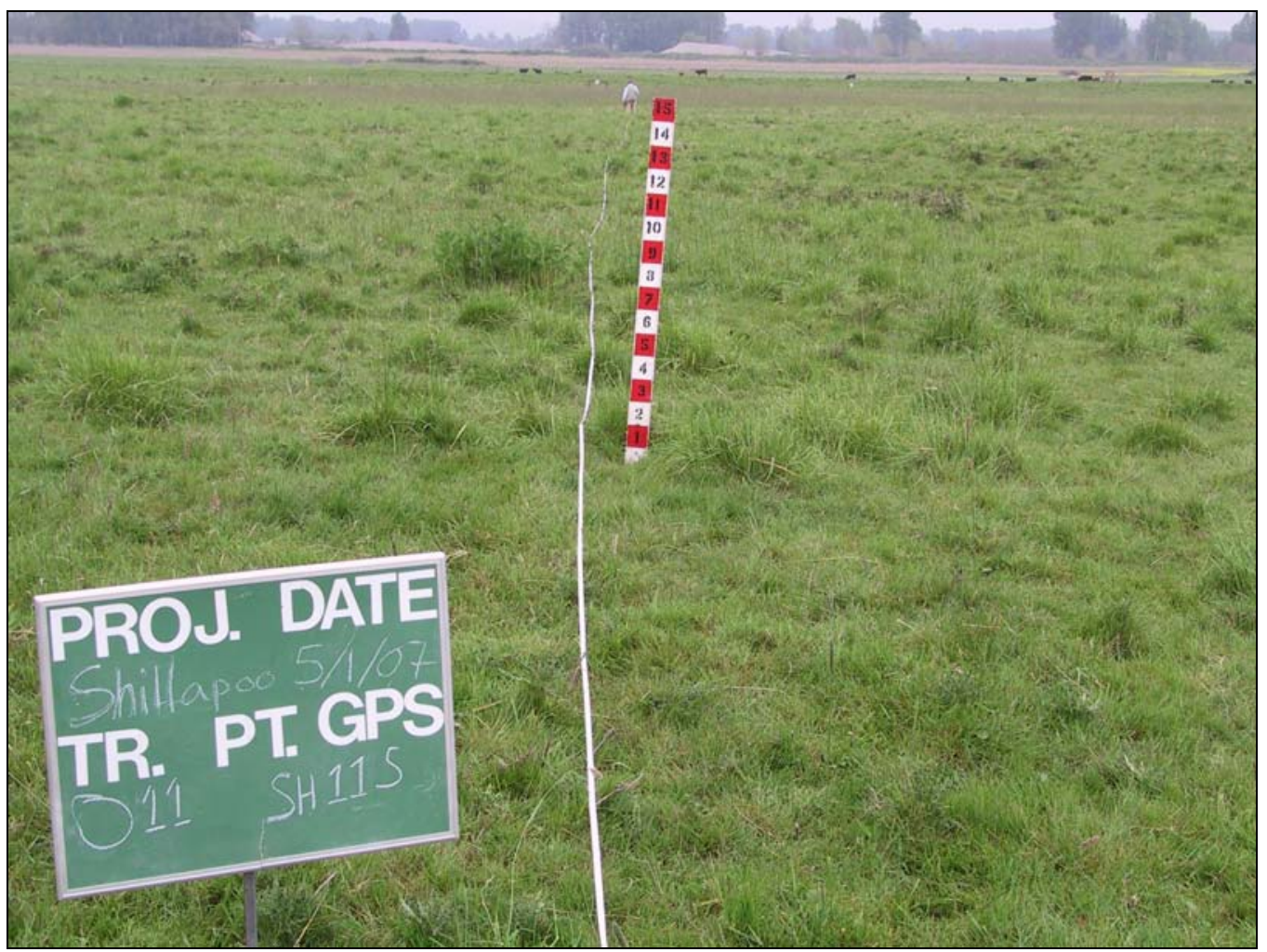


Shillapoo WA 2007 HEP Report

\section{Transect 12}

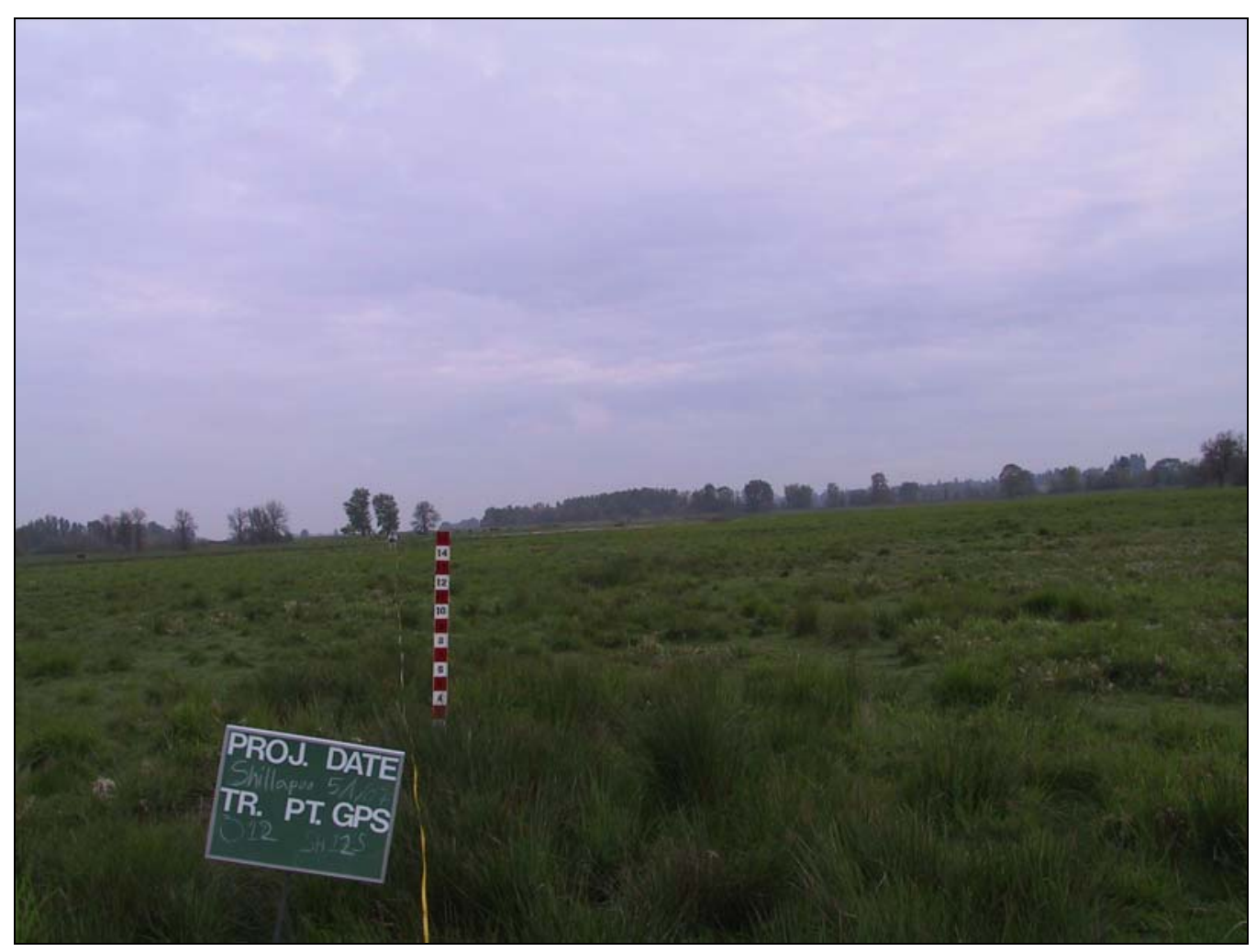

Transect 13

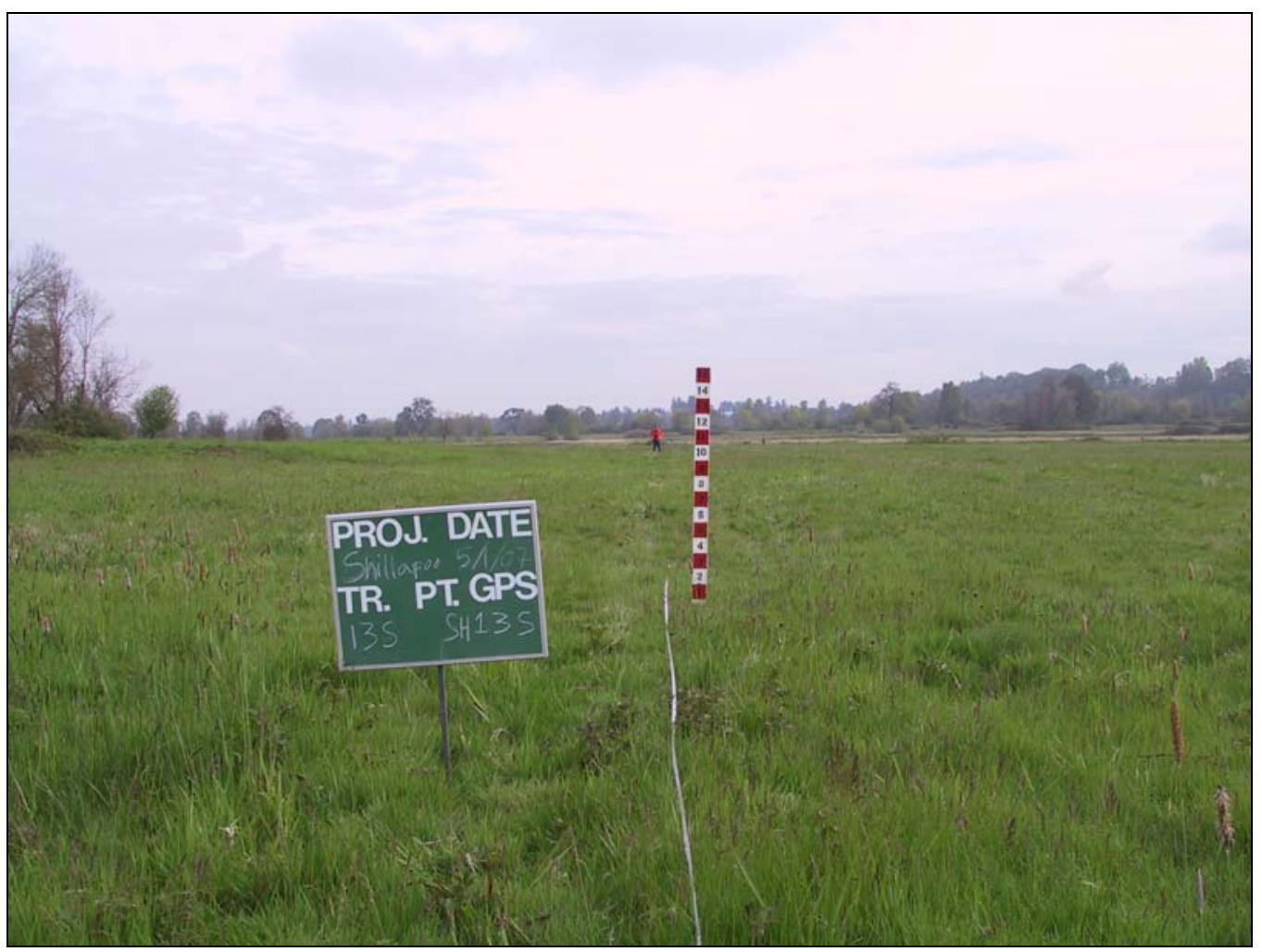


Shillapoo WA 2007 HEP Report

\section{Transect 14}

No Photograph Available

Transect 15

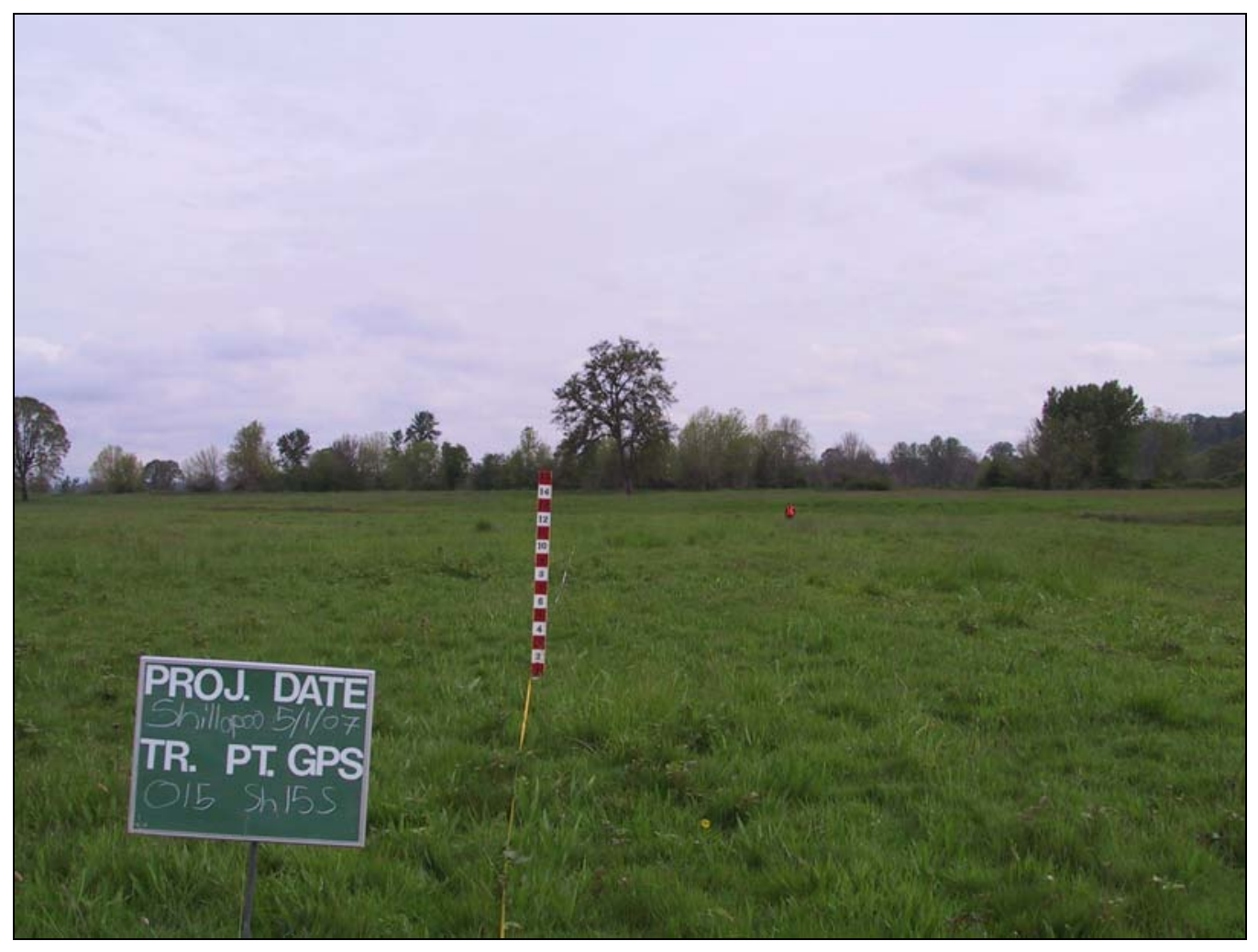


Shillapoo WA 2007 HEP Report

\section{Transect 16}

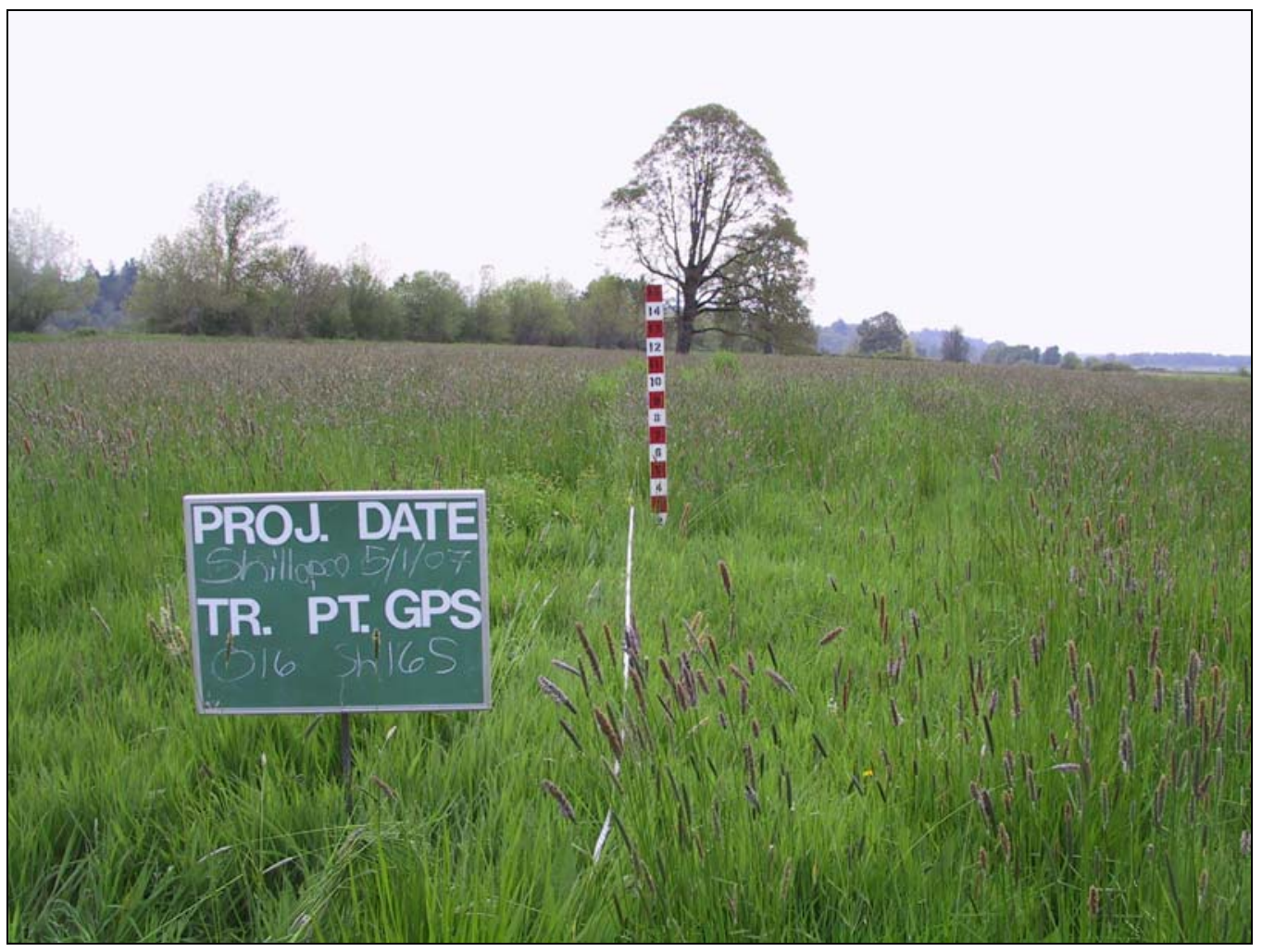

\section{Transect 17}

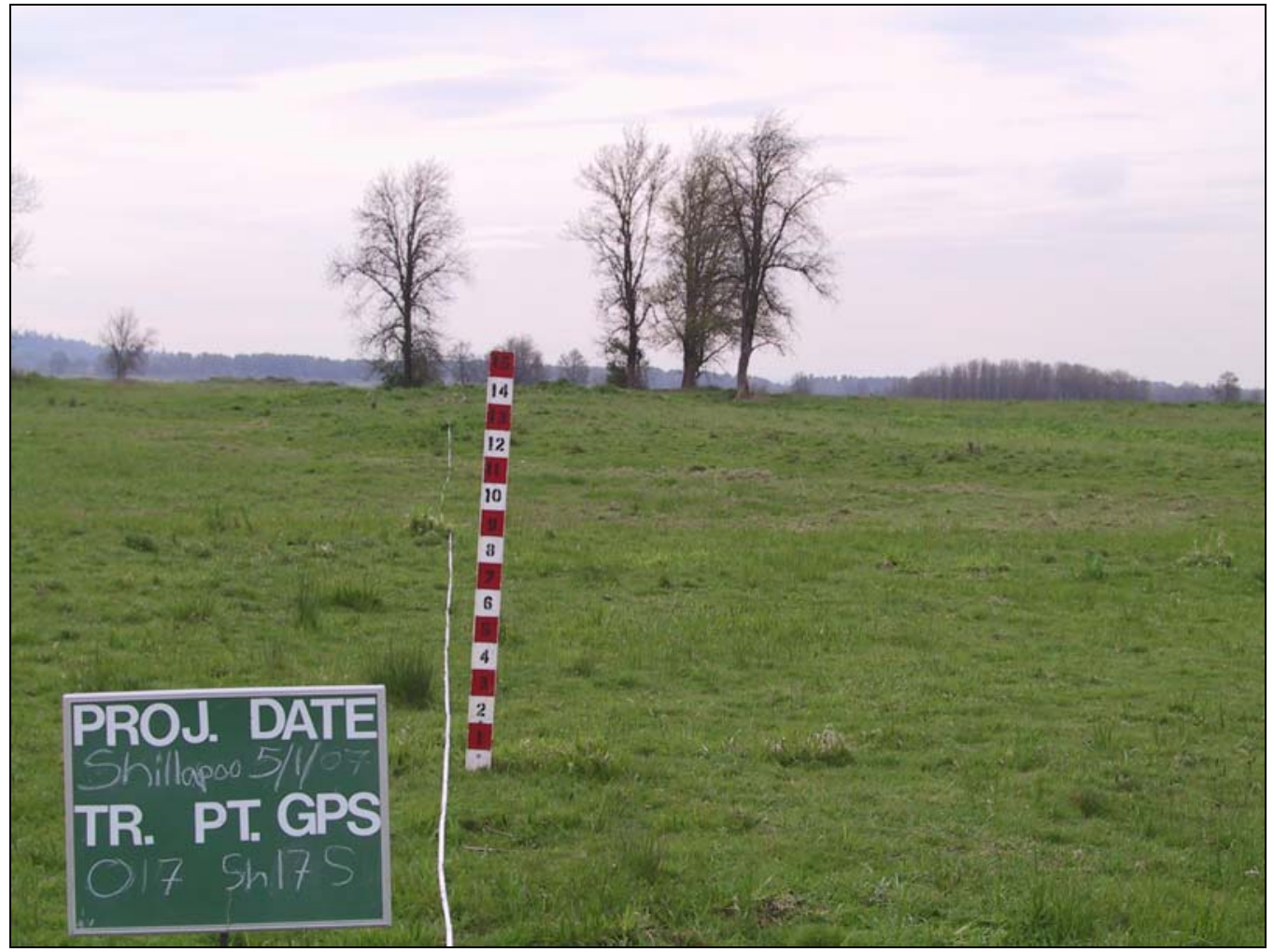


Shillapoo WA 2007 HEP Report

Transect 18

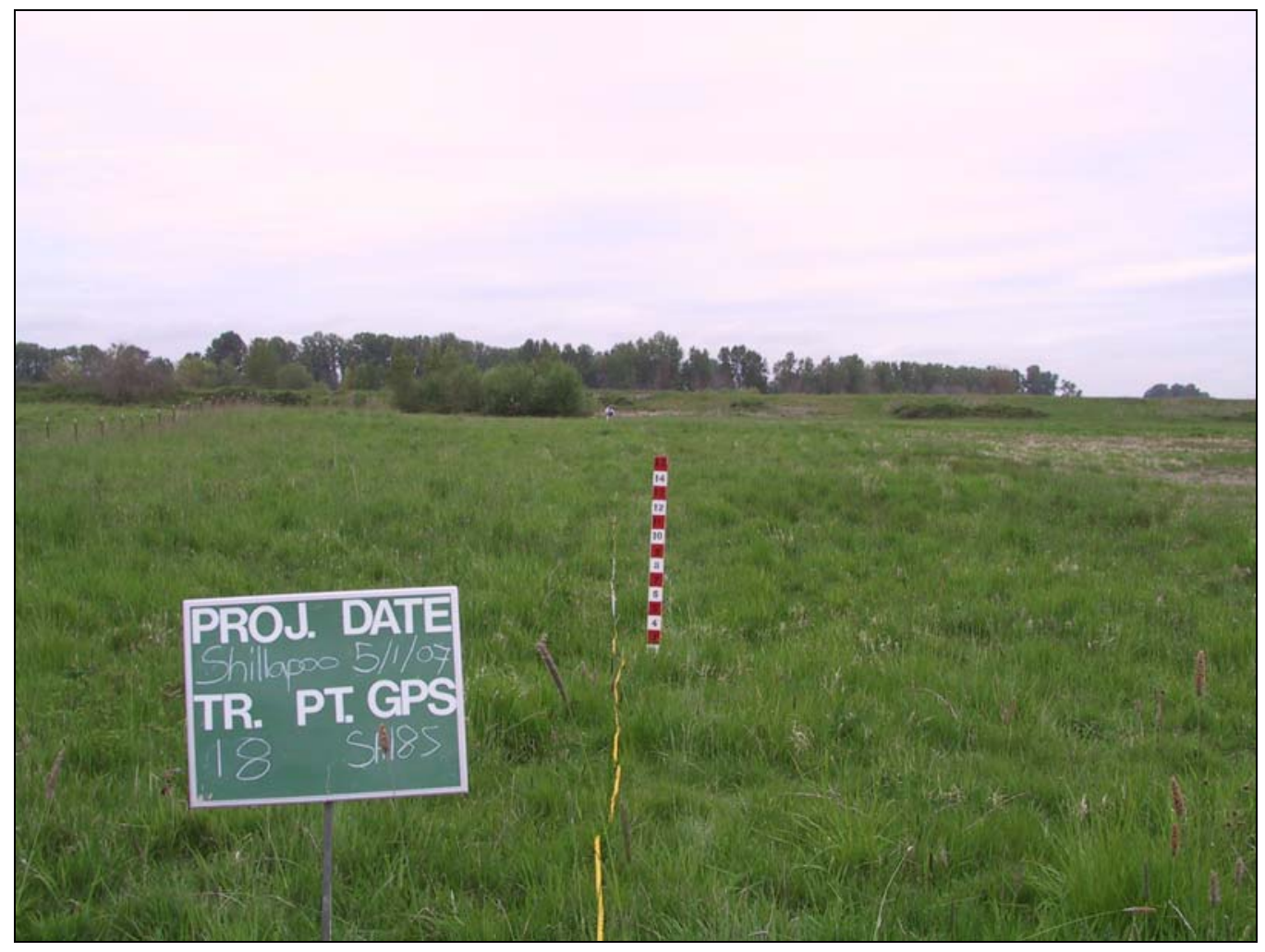

